Research Article

\title{
Existence and Uniqueness for a System of Caputo-Hadamard Fractional Differential Equations with Multipoint Boundary Conditions
}

\author{
S. Nageswara Rao $\left(\mathbb{D}\right.$, Ahmed Hussein Msmali $\mathbb{D}^{\mathbb{D}}$, Manoj Singh $(\mathbb{D}$, \\ and Abdullah Ali H. Ahmadini
}

Department of Mathematics, Faculty of Science, Jazan University, Jazan, Saudi Arabia

Correspondence should be addressed to S. Nageswara Rao; snrao@jazanu.edu.sa

Received 29 September 2020; Revised 14 November 2020; Accepted 21 November 2020; Published 23 December 2020

Academic Editor: Mitsuru Sugimoto

Copyright (c) 2020 S. Nageswara Rao et al. This is an open access article distributed under the Creative Commons Attribution License, which permits unrestricted use, distribution, and reproduction in any medium, provided the original work is properly cited.

\begin{abstract}
In this paper, we study existence and uniqueness of solutions for a system of Caputo-Hadamard fractional differential equations supplemented with multi-point boundary conditions. Our results are based on some classical fixed point theorems such as Banach contraction mapping principle, Leray-Schauder fixed point theorems. At last, we have presented two examples for the illustration of main results.
\end{abstract}

\section{Introduction}

In recent years, fractional differential equations (FDE) gain enormous attention among scientists due to the applications which were not possible with ordinary or partial differential equations of integer order. FDEs becomes a very successful tool in modeling anomalous diffusion and fractal-like nature. Agrawal discusses diffusion and heat equations of fractional order in [1-3]. Agrawal et al., Baleanu, and others investigated the boundary value problems for fractional differential equations [4]. Fractional dynamic models, fractional control systems, fractional population dynamics models, and fractional fluid dynamics all involve at least one ordinary or partial fractional derivative.

Fractional differential equations have several kinds of fractional derivatives, such as Riemann-Liouville fractional derivative, Caputo fractional derivative, and GrunwaldLetnikov fractional derivative. Another kind of fractional derivative is Hadamard type which was introduced in 1892 [5]. This derivative differs from various derivatives in the sense that the kernel of the integral in the definition of Hadamard derivative contains logarithmic function of arbitrary exponent. A detailed description of Hadamard fractional derivative and integral can be found in [6]. The readers who are interested in the subject of fractional calculus is referred to the books by Kilbas et al. [7], Podlubny [8], Miller and Ross [9], Samko et al. [10], Diethelm [11], and Zhou [12] and the references therein.

Coupled systems of fractional differential equations play a key role in developing differential models such as the synchronization of chaotic systems [13-15], anomalous diffusion $[16,17]$, disease models $[18,19]$, ecological models [20], Lorenz system [21], and nonlocal thermoelectricity systems $[22,23]$. For recent theoretical results on the topic, we refer the reader to a series of papers [24-37] and the references cited therein. Ahmad and Ntouyas $[32,33]$ discussed some fractional integral boundary value problems involving Hadamard fractional differential equations/systems and obtained the existence and uniqueness of solutions by applying the Banach fixed point theorem and Leray-Schauder alternative, respectively.

In [35], the authors investigated the existence and uniqueness of solutions for the coupled system of nonlinear fractional differential equations with three-point boundary conditions 


$$
\left\{\begin{array}{l}
\mathscr{D}^{\alpha} u(t)=f\left(t, v(t), \mathscr{D}^{p} v(t)\right), t \in(0,1), \\
\mathscr{D}^{\beta} v(t)=g\left(t, v(t), \mathscr{D}^{q} u(t)\right), t \in(0,1), \\
u(0)=0, u(1)=\gamma u(\eta), v(0)=0, v(1)=\gamma v(\eta),
\end{array}\right.
$$

where $1<\alpha, \beta<2, p, q, \gamma>0,0<\eta<1, \alpha-q \geq 1, \beta-p \geq 1, \gamma$ $\eta^{\alpha-1}<1, \gamma \eta^{\beta-1}<1$ and $\mathscr{D}^{\alpha}, \mathscr{D}^{\beta}$ are the standard RiemannLiouville fractional derivative and $f, g:[0,1] \times R \times R \rightarrow R$ are given continuous functions.

Recently, Alsulami et al. [36] established the existence and uniqueness results for a nonlinear coupled system of Caputo type fractional differential equations supplemented with nonseparated coupled boundary conditions.

$$
\left\{\begin{array}{l}
{ }^{c} \mathscr{D}^{\alpha} x(t)=f(t, x(t), y(t)), t \in[0, T], 1<\alpha \leq 2 \\
{ }^{c} \mathscr{D}^{\beta} y(t)=g(t, x(t), y(t)), t \in[0, T], 1<\beta \leq 2, \\
x(0)=\lambda_{1} y(T), x^{\prime}(0)=\lambda_{2} y^{\prime}(T), \\
y(0)=\mu_{1} y(T), y^{\prime}(0)=\mu_{2} x^{\prime}(T),
\end{array}\right.
$$

where ${ }^{c} \mathscr{D}^{\alpha}, \mathscr{D}^{\beta}$ denote the Caputo fractional derivatives of order $\alpha$ and $\beta$, respectively, $f, g:[0, T] \times R \times R \rightarrow R$ are appropriately chosen functions, and $\lambda_{i}, \mu_{i}, i=1,2$, are real constants with $\lambda_{i} \mu_{i} \neq 1, i=1,2$.

Motivated by the research going on in this direction, in this paper, we study existence and uniqueness of solutions for a coupled system of Caputo-Hadamard fractional differential equations.

$$
\left\{\begin{array}{l}
{ }^{c} \mathscr{D}_{a^{+}}^{\alpha} u(t)=f(t, u(t), v(t)), t \in[a, b], \\
{ }^{c} \mathscr{D}_{a^{+}}^{\beta} v(t)=g(t, u(t), v(t)), t \in[a, b]
\end{array}\right.
$$

with multipoint boundary conditions

$$
\left\{\begin{array}{l}
u(a)=\lambda_{1} v(b), \lambda_{2}{ }^{\mathscr{C}} \mathscr{D}_{a^{+}}^{\gamma_{1}} u(b)=\mu_{2} \sum_{i=1}^{N} \mathscr{C} \mathscr{D}_{a^{+}}^{\delta_{1}} v\left(\eta_{i}\right), \\
v(a)=\mu_{1} u(b), \lambda_{3}{ }^{\mathscr{C}} \mathscr{D}_{a^{+}}^{\gamma_{2}} v(b)=\mu_{3} \sum_{i=1}^{M}{ }^{\mathscr{C}} \mathscr{D}_{a^{+}}^{\delta_{2}} v\left(\xi_{i}\right),
\end{array}\right.
$$

where $\alpha, \beta \in(1,2], \gamma_{i}, \delta_{i} \in(0,1], i=1,2, \eta_{i} \in R$, for $i=1,2, \cdots$ $N(N \in N), a<\eta_{1}<\eta_{2}<\cdots<b, \xi_{i} \in R$, for $i=1,2, \cdots M(M \in$ $N), a<\xi_{1}<\xi_{2}<\cdots<b, \lambda_{i}, \mu_{i}, i=1,2,3$ are real positive constants $\mathscr{D}_{a^{+}}^{\kappa}$ denotes the Caputo-Hadamard fractional derivatives of order $\kappa$ for $\left(\kappa=\alpha, \beta, \gamma_{i}, \delta_{i}\right.$, for $\left.i=1,2\right)$, $f, g \in[a, b] \times R \times R \rightarrow R$ are appropriately chosen functions.

The paper is organized as follows. In Sect. 2, we present some preliminary concepts of fractional calculus. Sect. 3 contains main results concerning the existence and uniqueness of solutions for the given problem (3), (4). The LeraySchauder alternative theorem is applied to prove existence, while the uniqueness result was obtained via the Banach contraction mapping principle. Finally, we also discuss some examples for illustration of the existence-uniqueness results.

\section{Preliminaries}

For the convenience of the reader, we present some concepts of Hadamard type fractional calculus to facilitate the analysis of system (3), (4).

Definition 1 [7]. The Hadamard fractional integral of order $q>0$ of a function $x(t)$ for all $t>a>0$ is defined by

$$
{ }^{H} \mathscr{J}_{a^{+}}^{q} x(t)=\frac{1}{\Gamma(q)} \int_{a}^{t}\left(\ln \frac{t}{s}\right)^{q-1} x(s) \frac{d s}{s}
$$

where $\Gamma(q)=\int_{0}^{\infty} t^{q-1} e^{-t} d t$ is the gamma function,s provided the right side is pointwise defined on $R^{+}$.

Definition 2 [7]. The Hadamard fractional derivative of order $q>0$ of a function $x(t)$ for all $t>a>0$ is defined by

$$
{ }^{H} \mathscr{D}_{a^{+}}^{q} x(t)=\frac{1}{\Gamma(n-q)}\left(t \frac{d}{d t}\right)^{n} \int_{a}^{t}\left(\ln \frac{t}{s}\right)^{n-q-1} x(s) \frac{d s}{s},
$$

where $n=[q]+1$ with $[q]$ denotes the integral part of the real number $q$ and $\ln (\cdot)=\ln _{e}(\cdot)$.

Definition 3 [38]. Let $q \geq 0$ and $n=[q]+1$. If $y(x) \in A C_{\delta}^{n}[a, b]$, where $0<a<b<\infty$ and

$A C_{\delta}^{n}[a, b]=\left\{g:[a, b] \rightarrow C: \delta^{n-1} g(x) \in A C[a, b], \delta=x \frac{d}{d x}\right\}$

The Caputo type modification of the Hadamard fractional derivative of order $q$ is defined by

$$
{ }^{\mathscr{C}} \mathscr{D}_{a^{+}}^{q} y(x)=\mathscr{D}_{a^{+}}^{q}\left[y(t)-\sum_{k=0}^{n-1} \frac{\delta^{k} y(a)}{k !}\left(\ln \frac{t}{a}\right)^{k}\right](x) .
$$

Theorem 4 [38]. Let $q \geq 0$, and $n=[q]+1$. If $y(t) \in A C_{\delta}^{n}[a, b]$, where $0<a<b<\infty$. Then ${ }^{C} \mathscr{D}_{a^{+}}^{q} f(t)$ exist everywhere on $[a, b]$ and

(i) if $q \notin N_{0}, \mathscr{C}^{q} a_{a^{+}}^{q} f(t)$ can be represented by

$$
\mathscr{C}_{a^{+}}^{q} y(t)=\mathcal{F}_{a^{+}}^{n-q} \delta^{n} y(t)=\frac{1}{\Gamma(n-q)} \int_{a}^{t}\left(\ln \frac{t}{s}\right)^{n-q-1} \delta^{n} y(s) \frac{d s}{s},
$$

(ii) if $q \in N_{0}$, then ${ }^{\mathscr{C}} \mathscr{D}_{a^{+}}^{q} y(t)=\delta^{n} y(t)$

Remark 5. If $a, \alpha, \beta>0$ then

$$
\left({ }^{H} \mathscr{D}_{a}^{\alpha}\left(\ln \frac{t}{a}\right)^{\beta-1}\right)(x)=\frac{\Gamma(\beta)}{\Gamma(\beta-\alpha)}\left(\ln \frac{x}{a}\right)^{\beta-\alpha-1}
$$


Lemma 6 [38]. Let $q \geq 0$ and $n=[q]+1$. If $x(t) \in A C_{\delta}^{n}[a, b]$, then the Caputo-Hadamard fractional differential equation ${ }^{\mathscr{C}} \mathscr{D}_{a^{+}}^{q} x(t)=0$ has a solution:

$$
x(t)=\sum_{k=0}^{n-1} c_{k}\left(\log \frac{t}{a}\right)^{k}
$$

and the following formula holds:

$$
\mathscr{F}_{a^{+}}^{q} \mathscr{C} \mathscr{D}_{a^{+}}^{q} x(t)=x(t)+\sum_{k=0}^{n-1} c_{k}\left(\ln \frac{t}{a}\right)^{k}
$$

where $c_{k} \in R, k=1,2, \cdots, n-1$.

Now, we present an auxiliary lemma for boundary value problem of linear fractional differential equation with Caputo-Hadamard derivative.

Lemma 7. Let $\Delta=\left(\lambda_{2} \lambda_{3} / \Gamma\left(2-\gamma_{1}\right) \Gamma\left(2-\gamma_{2}\right)\right)(\ln (b / a))^{2-\gamma_{1}-\gamma_{2}}$ $-\mu_{2} \mu_{3} / \Gamma\left(2-\delta_{1}\right) \Gamma\left(2-\delta_{2}\right) \sum_{i=1}^{N}\left(\ln \left(\eta_{i} / a\right)\right)^{1-\delta_{1}} \sum_{i=1}^{M}\left(\ln \left(\xi_{i} l\right.\right.$ a) $)^{1-\delta_{2}} \neq 0$ and $\mu_{1} \lambda_{1} \neq 1$. Let $x, y \in A C_{n}^{\delta}[a, b]$. Then, the solution of the linear Caputo-Hadamard fractional differential system

$$
\left\{\begin{array}{l}
{ }^{c} \mathscr{D}_{a^{+}}^{\alpha} u(t)=x(t), t \in[a, b], 1<\alpha \leq 2, \\
{ }^{c} \mathscr{D}_{a^{+}}^{\beta} v(t)=y(t), t \in[a, b], 1<\beta \leq 2, \\
u(a)=\lambda_{1} v(b), \lambda_{2}^{c} \mathscr{D}_{a^{+}}^{\gamma_{1}} u(b)=\mu_{2} \sum_{i=1}^{N^{c}} \mathscr{D}_{a^{+}}^{\delta_{1}} v\left(\eta_{i}\right), \\
v(a)=\mu_{1} u(b), \lambda_{3}^{c} \mathscr{D}_{a^{+}}^{\gamma_{2}} v(b)=\mu_{3} \sum_{i=1}^{M^{c}} \mathscr{D}_{a^{+}}^{\delta_{2}} v\left(\xi_{i}\right),
\end{array}\right.
$$

is equivalent to the system of integral equations

$$
\begin{aligned}
u(t)=\frac{\mu_{3}}{\Delta}[ & \frac{\mu_{1} \mu_{2} \lambda_{1}(\ln (b / a)) \sum_{i=1}^{N}\left(\ln \left(\eta_{i} / a\right)\right)^{1-\delta_{1}}}{\Gamma\left(2-\delta_{1}\right)\left(1-\mu_{1} \lambda_{1}\right)} \\
& \left.+\frac{\lambda_{1} \lambda_{2}(\ln (b / a))^{2-\gamma_{1}}}{\Gamma\left(2-\gamma_{1}\right)\left(1-\mu_{1} \lambda_{1}\right)}+\frac{\mu_{2}(\ln (t / a)) \sum_{i=1}^{N}\left(\ln \left(\eta_{i} / a\right)\right)^{1-\delta_{1}}}{\Gamma\left(2-\delta_{1}\right)}\right] B_{3} \\
- & \frac{\lambda_{3}}{\Delta}\left[\frac{\mu_{1} \mu_{2} \lambda_{1}(\ln (b / a)) \sum_{i=1}^{N}\left(\ln \left(\eta_{i} / a\right)\right)^{1-\delta_{1}}}{\Gamma\left(2-\delta_{1}\right)\left(1-\mu_{1} \lambda_{1}\right)}+\frac{\lambda_{1} \lambda_{2}(\ln (b / a))^{2-\gamma_{1}}}{\Gamma\left(2-\gamma_{1}\right)\left(1-\mu_{1} \lambda_{1}\right)}\right. \\
& \left.+\frac{\mu_{2}(\ln (t / a)) \sum_{i=1}^{N}\left(\ln \left(\eta_{i} / a\right)\right)^{1-\delta_{1}}}{\Gamma\left(2-\delta_{1}\right)}\right] A_{3}+\frac{\mu_{2}}{\Delta}\left[\frac{\mu_{1} \lambda_{1} \lambda_{3}(\ln (b / a))^{2-\gamma_{2}}}{\Gamma\left(2-\gamma_{2}\right)\left(1-\mu_{1} \lambda_{1}\right)}\right. \\
& \left.+\frac{\lambda_{1} \mu_{3}(\ln (b / a)) \sum_{i=1}^{M}\left(\ln \left(\xi_{i} / a\right)\right)^{1-\delta_{2}}}{\Gamma\left(2-\delta_{2}\right)\left(1-\mu_{1} \lambda_{1}\right)}+\frac{\lambda_{3}(\ln (t / a))(\ln (b / a))^{1-\gamma_{2}}}{\Gamma\left(2-\gamma_{2}\right)}\right] A_{2} \\
- & \frac{\lambda_{2}}{\Delta}\left[\frac{\mu_{1} \lambda_{1} \lambda_{3}(\ln (b / a))^{2-\gamma_{2}}}{\Gamma\left(2-\gamma_{2}\right)\left(1-\mu_{1} \lambda_{1}\right)}+\frac{\lambda_{1} \mu_{3}(\ln (b / a)) \sum_{i=1}^{M}\left(\ln \left(\xi_{i} / a\right)\right)^{1-\delta_{2}}}{\Gamma\left(2-\delta_{2}\right)\left(1-\mu_{1} \lambda_{1}\right)}\right. \\
& \left.+\frac{\lambda_{3}(\ln (t / a))(\ln (b / a))^{1-\gamma_{2}}}{\Gamma\left(2-\gamma_{2}\right)}\right] B_{2}+\frac{\lambda_{1}}{1-\mu_{1} \lambda_{1}}\left(\mu_{1} B_{1}+A_{1}\right) \\
& +\int_{a}^{t} \frac{(\ln (t / s))^{\alpha-1}}{\Gamma(\alpha)} x(s) \frac{d s}{s},
\end{aligned}
$$

$$
\begin{aligned}
v(t)=\frac{\mu_{3}}{\Delta} & \frac{\mu_{1} \lambda_{1} \lambda_{2}(\ln (b / a))^{2-\gamma_{1}}}{\Gamma\left(2-\gamma_{1}\right)\left(1-\mu_{1} \lambda_{1}\right)}+\frac{\mu_{1} \mu_{2}(\ln (b / a)) \sum_{i=1}^{N}\left(\ln \left(\eta_{i} / a\right)\right)^{1-\delta_{1}}}{\Gamma\left(2-\delta_{1}\right)\left(1-\mu_{1} \lambda_{1}\right)} \\
& \left.+\frac{\lambda_{2}(\ln (t / a))(\ln (b / a))^{1-\gamma_{1}}}{\Gamma\left(2-\gamma_{1}\right)}\right] B_{3}-\frac{\lambda_{3}}{\Delta}\left[\frac{\mu_{1} \lambda_{1} \lambda_{2}(\ln (b / a))^{2-\gamma_{1}}}{\Gamma\left(2-\gamma_{1}\right)\left(1-\mu_{1} \lambda_{1}\right)}\right. \\
& \left.+\frac{\mu_{1} \mu_{2}(\ln (b / a)) \sum_{i=1}^{N}\left(\ln \left(\eta_{i} / a\right)\right)^{1-\delta_{1}}}{\Gamma\left(2-\delta_{1}\right)\left(1-\mu_{1} \lambda_{1}\right)}+\frac{\lambda_{2}(\ln (t / a))(\ln (b / a))^{1-\gamma_{1}}}{\Gamma\left(2-\gamma_{1}\right)}\right] A_{3} \\
+ & \frac{\mu_{2}}{\Delta}\left[\frac{\mu_{1} \mu_{3} \lambda_{1}(\ln (b / a)) \sum_{i=1}^{M}\left(\ln \left(\xi_{i} / a\right)\right)^{1-\delta_{2}}}{\Gamma\left(2-\delta_{2}\right)\left(1-\mu_{1} \lambda_{1}\right)}+\frac{\mu_{1} \lambda_{3}(\ln (b / a))^{2-\gamma_{2}}}{\Gamma\left(2-\gamma_{2}\right)\left(1-\mu_{1} \lambda_{1}\right)}\right. \\
& \left.+\frac{\left.\mu_{3}(\ln (t / a)) \sum_{i=1}^{M}\left(\ln \left(\xi_{i} / a\right)\right)^{1-\delta_{2}}\right] A_{2}}{\Gamma\left(2-\delta_{2}\right)}\right] A_{2} \\
- & \frac{\lambda_{2}}{\Delta}\left[\frac{\mu_{1} \mu_{3} \lambda_{1}(\ln (b / a)) \sum_{i=1}^{M}\left(\ln \left(\xi_{i} / a\right)\right)^{1-\delta_{2}}}{\Gamma\left(2-\delta_{2}\right)\left(1-\mu_{1} \lambda_{1}\right)}+\frac{\mu_{1} \lambda_{3}(\ln (b / a))^{2-\gamma_{2}}}{\Gamma\left(2-\gamma_{2}\right)\left(1-\mu_{1} \lambda_{1}\right)}\right. \\
& \left.+\frac{\mu_{3}(\ln (t / a)) \sum_{i=1}^{M}\left(\ln \left(\xi_{i} / a\right)\right)^{1-\delta_{2}}}{\Gamma\left(2-\delta_{2}\right)}\right] B_{2}+\frac{\mu_{1}}{1-\mu_{1} \lambda_{1}}\left(\lambda_{1} A_{1}+B_{1}\right) \\
+ & \int_{a}^{t} \frac{(\ln (t / s))^{\beta-1}}{\Gamma(\beta)} y(s) \frac{d s}{s},
\end{aligned}
$$

where

$$
\begin{aligned}
& B_{1}=\int_{a}^{b} \frac{(\ln (b / s))^{\alpha-1}}{\Gamma(\alpha)} x(s) \frac{d s}{s}, A_{1}=\int_{a}^{b} \frac{(\ln (b / s))^{\beta-1}}{\Gamma(\beta)} y(s) \frac{d s}{s}, \\
& B_{2}=\int_{a}^{b} \frac{(\ln (b / s))^{\alpha-\gamma_{1}-1}}{\Gamma\left(\alpha-\gamma_{1}\right)} x(s) \frac{d s}{s}, A_{2}=\sum_{i=1}^{N} \int_{a}^{\eta_{i}} \frac{\left(\ln \left(\eta_{i} / s\right)\right)^{\beta-\delta_{1}-1}}{\Gamma\left(\beta-\delta_{1}\right)} y(s) \frac{d s}{s}, \\
& B_{3}=\sum_{i=1}^{M} \int_{a}^{\xi_{i}} \frac{\left(\ln \left(\xi_{i} / s\right)\right)^{\alpha-\delta_{2}-1}}{\Gamma\left(\alpha-\delta_{2}\right)} x(s) \frac{d s}{s}, A_{3}=\int_{a}^{b} \frac{(\ln (b / s))^{\beta-\gamma_{2}-1}}{\Gamma\left(\beta-\gamma_{2}\right)} y(s) \frac{d s}{s} .
\end{aligned}
$$

Proof. We apply Lemma [6] that the general solution of the Caputo-Hadamard fractional differential equation in (13) can be written as:

$$
\begin{gathered}
u(t)=c_{0}+c_{1}\left(\ln \frac{t}{a}\right)+\int_{a}^{t} \frac{(\ln (t / s))^{\alpha-1}}{\Gamma(\alpha)} x(s) \frac{d s}{s}, \\
v(t)=d_{0}+d_{1}\left(\ln \frac{t}{a}\right)+\int_{a}^{t} \frac{(\ln (t / s))^{\beta-1}}{\Gamma(\beta)} y(s) \frac{d s}{s},
\end{gathered}
$$

where $c_{i}, d_{i}, i=0,1$, are arbitrary real constants. From (17) and (18) we have

$$
\begin{aligned}
& { }_{D_{a^{+}}}^{\gamma_{1}} u(t)=c_{1} \frac{1}{\Gamma\left(2-\gamma_{1}\right)}\left(\ln \frac{t}{a}\right)^{1-\gamma_{1}}+\int_{a}^{t} \frac{(\ln (t / s))^{\alpha-\gamma_{1}-1}}{\Gamma\left(\alpha-\gamma_{1}\right)} x(s) \frac{d s}{s}, \\
& { }^{c} \mathscr{D}_{a^{+}}^{\gamma_{2}} v(t)=d_{1} \frac{1}{\Gamma\left(2-\gamma_{2}\right)}\left(\ln \frac{t}{a}\right)^{1-\gamma_{2}}+\int_{a}^{t} \frac{(\ln (t / s))^{\beta-\gamma_{2}-1}}{\Gamma\left(\beta-\gamma_{2}\right)} y(s) \frac{d s}{s}, \\
& { }^{c} \mathscr{D}_{a^{+}}^{\delta_{1}} v(t)=d_{1} \frac{1}{\Gamma\left(2-\delta_{1}\right)}\left(\ln \frac{t}{a}\right)^{1-\delta_{1}}+\int_{a}^{t} \frac{(\ln (t / s))^{\beta-\delta_{1}-1}}{\Gamma\left(\beta-\delta_{1}\right)} y(s) \frac{d s}{s},
\end{aligned}
$$


${ }^{c} \mathscr{D}_{a^{+}}^{\delta_{2}} u(t)=c_{1} \frac{1}{\Gamma\left(2-\delta_{2}\right)}\left(\ln \frac{t}{a}\right)^{1-\delta_{2}}+\int_{a}^{t} \frac{(\ln (t / s))^{\alpha-\delta_{2}-1}}{\Gamma\left(\alpha-\delta_{2}\right)} x(s) \frac{d s}{s}$.

Using the boundary conditions $u(a)=\lambda_{1} v(b)$ and $v(a)$ $=\mu_{1} u(b)$ from (17) and (18), we have

$$
\begin{aligned}
& \Rightarrow c_{0}=\lambda_{1}\left[d_{0}+d_{1}\left(\ln \frac{b}{a}\right)+A_{1}\right], \\
& \Rightarrow d_{0}=\mu_{1}\left[c_{0}+c_{1}\left(\ln \frac{b}{a}\right)+B_{1}\right] .
\end{aligned}
$$

Using the boundary conditions $\lambda_{2}{ }^{C} \mathscr{D}_{a^{+}}^{\gamma_{1}} u(b)=\mu_{2} \sum_{i=1}^{N}$ ${ }^{C} \mathscr{D}_{a^{+}}^{\delta_{1}} v\left(\eta_{i}\right)$ and $\lambda_{3}{ }^{\mathscr{C}} \mathscr{D}_{a^{+}}^{\gamma_{2}} v(b)=\mu_{3} \sum_{i=1}^{M} \mathscr{C} \mathscr{D}_{a^{+}}^{\delta_{2}} u\left(\xi_{i}\right)$ from (19) to (22), we have

$$
\begin{aligned}
& \Rightarrow c_{1} \frac{\lambda_{2}}{\Gamma\left(2-\gamma_{1}\right)}\left(\ln \frac{b}{a}\right)^{1-\gamma_{1}}-d_{1} \frac{\mu_{2}}{\Gamma\left(2-\delta_{1}\right)} \sum_{i=1}^{N}\left(\ln \frac{\eta_{i}}{a}\right)^{1-\delta_{1}}=A_{2} \mu_{2}-\lambda_{2} B_{2}, \\
& \Rightarrow c_{1} \frac{-\mu_{3}}{\Gamma\left(2-\delta_{2}\right)} \sum_{i=1}^{M}\left(\ln \frac{\xi_{i}}{a}\right)^{1-\delta_{2}}+d_{1} \frac{\lambda_{3}}{\Gamma\left(2-\gamma_{2}\right)}\left(\ln \frac{b}{a}\right)^{1-\gamma_{2}}=B_{3} \mu_{3}-\lambda_{3} A_{3} .
\end{aligned}
$$

Solving the resulting equations for $c_{1}$ and $d_{1}$, we find that $c_{1}=\frac{1}{\Delta}\left[\frac{\lambda_{3}\left(A_{2} \mu_{2}-\lambda_{2} B_{2}\right)}{\Gamma\left(2-\gamma_{2}\right)}\left(\ln \frac{b}{a}\right)^{1-\gamma_{2}}+\frac{\mu_{2}\left(B_{3} \mu_{3}-\lambda_{3} A_{3}\right)}{\Gamma\left(2-\delta_{1}\right)} \sum_{i=1}^{N}\left(\ln \frac{\eta_{i}}{a}\right)^{1-\delta_{1}}\right]$, $d_{1}=\frac{1}{\Delta}\left[\frac{\lambda_{2}\left(B_{3} \mu_{3}-\lambda_{3} A_{3}\right)}{\Gamma\left(2-\gamma_{1}\right)}\left(\ln \frac{b}{a}\right)^{1-\gamma_{1}}+\frac{\mu_{3}\left(A_{2} \mu_{2}-\lambda_{2} B_{2}\right)}{\Gamma\left(2-\delta_{2}\right)} \sum_{i=1}^{M}\left(\ln \frac{\xi_{i}}{a}\right)^{1-\delta_{2}}\right]$,

substituting $c_{1}$ and $d_{1}$ in (23) and (24), we have

$$
\begin{aligned}
c_{0}= & \frac{\lambda_{1}}{1-\mu_{1} \lambda_{1}}\left[\left[\frac{\mu_{1} \mu_{2} \mu_{3}}{\Delta \Gamma\left(2-\delta_{1}\right)} \sum_{i=1}^{N}\left(\ln \frac{\eta_{i}}{a}\right)^{1-\delta_{1}}\left(\ln \frac{b}{a}\right)+\frac{\lambda_{2} \mu_{3}}{\Delta \Gamma\left(2-\gamma_{1}\right)}\right.\right. \\
& \left.\cdot\left(\ln \frac{b}{a}\right)^{2-\gamma_{1}}\right] B_{3}-\left[\frac{\mu_{1} \mu_{2} \lambda_{3}}{\Delta \Gamma\left(2-\delta_{1}\right)} \sum_{i=1}^{N}\left(\ln \frac{\eta_{i}}{a}\right)^{1-\delta_{1}}\left(\ln \frac{b}{a}\right)\right. \\
& \left.+\frac{\lambda_{2} \lambda_{3}}{\Delta \Gamma\left(2-\gamma_{1}\right)}\left(\ln \frac{b}{a}\right)^{2-\gamma_{1}}\right] A_{3}+\left[\frac{\mu_{1} \mu_{2} \lambda_{3}}{\Delta \Gamma\left(2-\gamma_{2}\right)}\left(\ln \frac{b}{a}\right)^{2-\gamma_{2}}\right. \\
& \left.+\frac{\mu_{2} \mu_{3}}{\Delta \Gamma\left(2-\delta_{2}\right)} \sum_{i=1}^{M}\left(\ln \frac{\xi_{i}}{a}\right)^{1-\delta_{2}}\left(\ln \frac{b}{a}\right)\right] A_{2} \\
& -\left[\frac{\mu_{1} \lambda_{2} \lambda_{3}}{\Delta \Gamma\left(2-\gamma_{2}\right)}\left(\ln \frac{b}{a}\right)^{2-\gamma_{2}}+\frac{\lambda_{2} \mu_{3}}{\Delta \Gamma\left(2-\delta_{2}\right)} \sum_{i=1}^{M}\left(\ln \frac{\xi_{i}}{a}\right)^{1-\delta_{2}}\right. \\
& \left.\left.\cdot\left(\ln \frac{b}{a}\right)\right] B_{2}+\left(\mu_{1} B_{1}+A_{1}\right)\right],
\end{aligned}
$$

and

$$
\begin{aligned}
d_{0}= & \frac{\lambda_{1}}{1-\mu_{1} \lambda_{1}}\left[\left[\frac{\lambda_{1} \lambda_{2} \mu_{3}}{\Delta \Gamma\left(2-\gamma_{1}\right)}\left(\ln \frac{b}{a}\right)^{2-\gamma_{1}}+\frac{\mu_{2} \mu_{3}}{\Delta \Gamma\left(2-\delta_{1}\right)} \sum_{i=1}^{N}\right.\right. \\
& \left.\cdot\left(\ln \frac{\eta_{i}}{a}\right)^{1-\delta_{1}}\left(\ln \frac{b}{a}\right)\right] B_{3}-\left[\frac{\lambda_{1} \lambda_{2} \lambda_{3}}{\Delta \Gamma\left(2-\gamma_{1}\right)}\left(\ln \frac{b}{a}\right)^{2-\gamma_{1}}\right. \\
& \left.+\frac{\mu_{2} \lambda_{3}}{\Delta \Gamma\left(2-\delta_{1}\right)} \sum_{i=1}^{N}\left(\ln \frac{\eta_{i}}{a}\right)^{1-\delta_{1}}\left(\ln \frac{b}{a}\right)\right] A_{3}+\left[\frac{\lambda_{1} \mu_{2} \mu_{3}}{\Delta \Gamma\left(2-\delta_{2}\right)}\right. \\
& \left.\cdot \sum_{i=1}^{M}\left(\ln \frac{\xi_{i}}{a}\right)^{1-\delta_{2}}\left(\ln \frac{b}{a}\right)+\frac{\mu_{2} \lambda_{3}}{\Delta \Gamma\left(2-\gamma_{2}\right)}\left(\ln \frac{b}{a}\right)^{2-\gamma_{2}}\right] A_{2} \\
& -\left[\frac{\lambda_{1} \lambda_{2} \mu_{3}}{\Delta \Gamma\left(2-\delta_{2}\right)} \sum_{i=1}^{M}\left(\ln \frac{\xi_{i}}{a}\right)^{1-\delta_{2}}\left(\ln \frac{b}{a}\right)+\frac{\lambda_{2} \lambda_{3}}{\Delta \Gamma\left(2-\gamma_{2}\right)}\right. \\
& \left.\left.\cdot\left(\ln \frac{b}{a}\right)^{2-\gamma_{2}}\right] B_{2}+\left(\lambda_{1} A_{1}+B_{1}\right)\right] .
\end{aligned}
$$

Inserting the values of $c_{i}, d_{i}, i=0,1$ in (17) and (18), which leads to the solution system (14), (15). The converse follows by direct computation. The proof is completed.

\section{Existence and Uniqueness Results}

This section is concerned with the main results of the paper.

First of all, we fix our terminology. Let $\mathscr{C}=C([a, b], R), a>0$ be the Banach space of all continuous functions from $[a, b]$ to $R$. Space $X=\left\{u(t): u(t) \in C^{2}([a, b], R)\right\}$ endowed with the norm $\|u\|=\sup \{|u(t)|, t \in[a, b]\}$ is a Banach space. In addition, let $Y=\left\{v(t): v(t) \in C^{2}([a, b], R)\right\}$ with the norm $\|v\|=\sup \{\mid v($ $t) \mid, t \in[a, b]\}$. It is obvious that product space $(X \times Y, \|(u, v)$ I) $)$ is a Banach space with the norm $\|(u, v)\|=\|u\|+\|v\|$.

In view of Lemma 7 , we introduce an operator $\mathscr{T}: X \times$ $Y \rightarrow X \times Y$ as follows:

$$
\mathscr{T}(u, v)(t)=\left(\mathscr{T}_{1}(u, v)(t), \mathscr{T}_{2}(u, v)(t)\right),
$$

where

$$
\begin{aligned}
\mathscr{T}_{1}(u, v)(t)= & \frac{\mu_{3}}{\Delta}\left[\frac{\mu_{1} \mu_{2} \lambda_{1}(\ln (b / a)) \sum_{i=1}^{N}\left(\ln \left(\eta_{i} / a\right)\right)^{1-\delta_{1}}}{\Gamma\left(2-\delta_{1}\right)\left(1-\mu_{1} \lambda_{1}\right)}\right. \\
& \left.+\frac{\lambda_{1} \lambda_{2}(\ln (b / a))^{2-\gamma_{1}}}{\Gamma\left(2-\gamma_{1}\right)\left(1-\mu_{1} \lambda_{1}\right)}+\frac{\mu_{2}(\ln (t / a)) \sum_{i=1}^{N}\left(\ln \left(\eta_{i} / a\right)\right)^{1-\delta_{1}}}{\Gamma\left(2-\delta_{1}\right)}\right] B_{3 f} \\
& -\frac{\lambda_{3}}{\Delta}\left[\frac{\mu_{1} \mu_{2} \lambda_{1}(\ln (b / a)) \sum_{i=1}^{N}\left(\ln \left(\eta_{i} / a\right)\right)^{1-\delta_{1}}}{\Gamma\left(2-\delta_{1}\right)\left(1-\mu_{1} \lambda_{1}\right)}+\frac{\lambda_{1} \lambda_{2}(\ln (b / a))^{2-\gamma_{1}}}{\Gamma\left(2-\gamma_{1}\right)\left(1-\mu_{1} \lambda_{1}\right)}\right. \\
& \left.+\frac{\mu_{2}(\ln (t / a)) \sum_{i=1}^{N}\left(\ln \left(\eta_{i} / a\right)\right)^{1-\delta_{1}}}{\Gamma\left(2-\delta_{1}\right)}\right] A_{3 g}+\frac{\mu_{2}}{\Delta}\left[\frac{\mu_{1} \lambda_{1} \lambda_{3}(\ln (b / a))^{2-\gamma_{2}}}{\Gamma\left(2-\gamma_{2}\right)\left(1-\mu_{1} \lambda_{1}\right)}\right. \\
& \left.+\frac{\lambda_{1} \mu_{3}(\ln (b / a)) \sum_{i=1}^{M}\left(\ln \left(\xi_{i} / a\right)\right)^{1-\delta_{2}}}{\Gamma\left(2-\delta_{2}\right)\left(1-\mu_{1} \lambda_{1}\right)}+\frac{\lambda_{3}(\ln (t / a))(\ln (b / a))^{1-\gamma_{2}}}{\Gamma\left(2-\gamma_{2}\right)}\right] A_{2 g} \\
& -\frac{\lambda_{2}}{\Delta}\left[\frac{\mu_{1} \lambda_{1} \lambda_{3}(\ln (b / a))^{2-\gamma_{2}}}{\Gamma\left(2-\gamma_{2}\right)\left(1-\mu_{1} \lambda_{1}\right)}+\frac{\lambda_{1} \mu_{3}(\ln (b / a)) \sum_{i=1}^{M}\left(\ln \left(\xi_{i} / a\right)\right)^{1-\delta_{2}}}{\Gamma\left(2-\delta_{2}\right)\left(1-\mu_{1} \lambda_{1}\right)}\right. \\
& \left.+\frac{\lambda_{3}(\ln (t / a))(\ln (b / a))^{1-\gamma_{2}}}{\Gamma\left(2-\gamma_{2}\right)}\right] B_{2 f}+\frac{\lambda_{1}}{1-\mu_{1} \lambda_{1}}\left(\mu_{1} B_{1 f}+A_{1 g}\right) \\
& +\int_{a}^{t} \frac{(\ln (t / s))^{\alpha-1}}{\Gamma(\alpha)} f(s, u(s), v(s)) \frac{d s}{s},
\end{aligned}
$$


and

$$
\begin{aligned}
\mathscr{T}_{2}(u, v)(t)= & \frac{\mu_{3}}{\Delta}\left[\frac{\mu_{1} \lambda_{1} \lambda_{2}(\ln (b / a))^{2-\gamma_{1}}}{\Gamma\left(2-\gamma_{1}\right)\left(1-\mu_{1} \lambda_{1}\right)}+\frac{\mu_{1} \mu_{2}(\ln (b / a)) \sum_{i=1}^{N}\left(\ln \left(\eta_{i} / a\right)\right)^{1-\delta_{1}}}{\Gamma\left(2-\delta_{1}\right)\left(1-\mu_{1} \lambda_{1}\right)}\right. \\
& \left.+\frac{\lambda_{2}(\ln (t / a))(\ln (b / a))^{1-\gamma_{1}}}{\Gamma\left(2-\gamma_{1}\right)}\right] B_{3 f}-\frac{\lambda_{3}}{\Delta}\left[\frac{\mu_{1} \lambda_{1} \lambda_{2}(\ln (b / a))^{2-\gamma_{1}}}{\Gamma\left(2-\gamma_{1}\right)\left(1-\mu_{1} \lambda_{1}\right)}\right. \\
& \left.+\frac{\mu_{1} \mu_{2}(\ln (b / a)) \sum_{i=1}^{N}\left(\ln \left(\eta_{i} / a\right)\right)^{1-\delta_{1}}}{\Gamma\left(2-\delta_{1}\right)\left(1-\mu_{1} \lambda_{1}\right)}+\frac{\lambda_{2}(\ln (t / a))(\ln (b / a))^{1-\gamma_{1}}}{\Gamma\left(2-\gamma_{1}\right)}\right] A_{3 g} \\
& +\frac{\mu_{2}}{\Delta}\left[\frac{\mu_{1} \mu_{3} \lambda_{1}(\ln (b / a)) \sum_{i=1}^{M}\left(\ln \left(\xi_{i} / a\right)\right)^{1-\delta_{2}}}{\Gamma\left(2-\delta_{2}\right)\left(1-\mu_{1} \lambda_{1}\right)}+\frac{\mu_{1} \lambda_{3}(\ln (b / a))^{2-\gamma_{2}}}{\Gamma\left(2-\gamma_{2}\right)\left(1-\mu_{1} \lambda_{1}\right)}\right. \\
& \left.+\frac{\mu_{3}(\ln (t / a)) \sum_{i=1}^{M}\left(\ln \left(\xi_{i} / a\right)\right)^{1-\delta_{2}}}{\Gamma\left(2-\delta_{2}\right)}\right] A_{2 g} \\
& -\frac{\lambda_{2}}{\Delta}\left[\frac{\mu_{1} \mu_{3} \lambda_{1}(\ln (b / a)) \sum_{i=1}^{M}\left(\ln \left(\xi_{i} / a\right)\right)^{1-\delta_{2}}}{\Gamma\left(2-\delta_{2}\right)\left(1-\mu_{1} \lambda_{1}\right)}+\frac{\mu_{1} \lambda_{3}(\ln (b / a))^{2-\gamma_{2}}}{\Gamma\left(2-\gamma_{2}\right)\left(1-\mu_{1} \lambda_{1}\right)}\right. \\
& \left.+\frac{\mu_{3}(\ln (t / a)) \sum_{i=1}^{M}\left(\ln \left(\xi_{i} / a\right)\right)^{1-\delta_{2}}}{\Gamma\left(2-\delta_{2}\right)}\right] B_{2 f}+\frac{\mu_{1}}{1-\mu_{1} \lambda_{1}}\left(\lambda_{1} A_{1 g}+B_{1 f}\right) \\
& +\int_{a}^{t} \frac{(\ln (t / s))^{\beta-1}}{\Gamma(\beta)} g(s, u(s), v(s)) \frac{d s}{s} .
\end{aligned}
$$

Here,

$$
\begin{aligned}
B_{1 f} & =\int_{a}^{b} \frac{(\ln (b / s))^{\alpha-1}}{\Gamma(\alpha)} f(s, u(s), v(s)) \frac{d s}{s}, A_{1 g} \\
& =\int_{a}^{b} \frac{(\ln (b / s))^{\beta-1}}{\Gamma(\beta)} g(s, u(s), v(s)) \frac{d s}{s}, \\
B_{2 f} & =\int_{a}^{b} \frac{(\ln (b / s))^{\alpha-\gamma_{1}-1}}{\Gamma\left(\alpha-\gamma_{1}\right)} f(s, u(s), v(s)) \frac{d s}{s}, A_{2 g} \\
& =\sum_{i=1}^{N} \int_{a}^{\eta_{i}} \frac{\left(\ln \left(\eta_{i} / s\right)\right)^{\beta-\delta_{1}-1}}{\Gamma\left(\beta-\delta_{1}\right)} g(s, u(s), v(s)) \frac{d s}{s}, \\
B_{3 f}= & \sum_{i=1}^{M} \int_{a}^{\xi_{i}} \frac{\left(\ln \left(\xi_{i} / s\right)\right)^{\alpha-\delta_{2}-1}}{\Gamma\left(\alpha-\delta_{2}\right)} f(s, u(s), v(s)) \frac{d s}{s}, A_{3 g} \\
= & \int_{a}^{b} \frac{(\ln (b / s))^{\beta-\gamma_{2}-1}}{\Gamma\left(\beta-\gamma_{2}\right)} g(s, u(s), v(s)) \frac{d s}{s} .
\end{aligned}
$$

For computational convenience, we set

$$
\begin{aligned}
K_{1}= & \frac{\left|\mu_{3}\right|}{|\Delta|}\left[\frac{\left|\mu_{1}\right|\left|\mu_{2}\right|\left|\lambda_{1}\right|(\ln (b / a)) \sum_{i=1}^{N}\left(\ln \left(\eta_{i} / a\right)\right)^{1-\delta_{1}}}{\Gamma\left(2-\delta_{1}\right)\left|1-\mu_{1} \lambda_{1}\right|}\right. \\
& \left.+\frac{\left|\lambda_{1}\right|\left|\lambda_{2}\right|(\ln (b / a))^{2-\gamma_{1}}}{\Gamma\left(2-\gamma_{1}\right)\left|1-\mu_{1} \lambda_{1}\right|}+\frac{\left|\mu_{2}\right|(\ln (b / a)) \sum_{i=1}^{N}\left(\ln \left(\eta_{i} / a\right)\right)^{1-\delta_{1}}}{\Gamma\left(2-\delta_{1}\right)}\right] \\
& \times \frac{\sum_{i=1}^{M}\left(\ln \left(\xi_{i} / a\right)\right)^{\alpha-\delta_{2}}}{\Gamma\left(\alpha-\delta_{2}+1\right)}+\frac{\left|\lambda_{2}\right|}{|\Delta|}\left[\frac{\left|\mu_{1}\right|\left|\lambda_{1}\right|\left|\lambda_{3}\right|(\ln (b / a))^{2-\gamma_{2}}}{\Gamma\left(2-\gamma_{2}\right)\left|1-\mu_{1} \lambda_{1}\right|}\right. \\
& \left.+\frac{\left|\lambda_{1}\right|\left|\mu_{3}\right|(\ln (b / a)) \sum_{i=1}^{M}\left(\ln \left(\xi_{i} / a\right)\right)^{1-\delta_{2}}}{\Gamma\left(2-\delta_{2}\right)\left|1-\mu_{1} \lambda_{1}\right|}+\frac{\left|\lambda_{3}\right|(\ln (b / a))^{2-\gamma_{2}}}{\Gamma\left(2-\gamma_{2}\right)}\right] \\
& \times \frac{(\ln (b / a))^{\alpha-\gamma_{1}}}{\Gamma\left(\alpha-\gamma_{1}+1\right)}+\left[\frac{\left|\mu_{1}\right|\left|\lambda_{1}\right|}{\left|1-\mu_{1} \lambda_{1}\right|}+1\right] \frac{(\ln (b / a))^{\alpha}}{\Gamma(\alpha+1)},
\end{aligned}
$$

$$
\begin{aligned}
& K_{2}=\frac{\left|\lambda_{3}\right|}{|\Delta|}\left[\frac{\left|\mu_{1}\right|\left|\mu_{2}\right|\left|\lambda_{1}\right|(\ln (b / a)) \sum_{i=1}^{N}\left(\ln \left(\eta_{i} / a\right)\right)^{1-\delta_{1}}}{\Gamma\left(2-\delta_{1}\right)\left|1-\mu_{1} \lambda_{1}\right|}\right. \\
& \left.+\frac{\left|\lambda_{1}\right|\left|\lambda_{2}\right|(\ln (b / a))^{2-\gamma_{1}}}{\Gamma\left(2-\gamma_{1}\right)\left|1-\mu_{1} \lambda_{1}\right|}+\frac{\left|\mu_{2}\right|(\ln (b / a)) \sum_{i=1}^{N}\left(\ln \left(\eta_{i} / a\right)\right)^{1-\delta_{1}}}{\Gamma\left(2-\delta_{1}\right)}\right] \\
& \times \frac{(\ln (b / a))^{\beta-\gamma_{2}}}{\Gamma\left(\beta-\gamma_{2}+1\right)}+\frac{\left|\mu_{2}\right|}{|\Delta|}\left[\frac{\left|\mu_{1}\right|\left|\lambda_{1}\right|\left|\lambda_{3}\right|(\ln (b / a))^{2-\gamma_{2}}}{\Gamma\left(2-\gamma_{2}\right)\left|1-\mu_{1} \lambda_{1}\right|}\right. \\
& \left.+\frac{\left|\lambda_{1}\right|\left|\mu_{3}\right|(\ln (b / a)) \sum_{i=1}^{M}\left(\ln \left(\xi_{i} / a\right)\right)^{1-\delta_{2}}}{\Gamma\left(2-\delta_{2}\right)\left|\left(1-\mu_{1} \lambda_{1}\right)\right|}+\frac{\left|\lambda_{3}\right|(\ln (b / a))^{2-\gamma_{2}}}{\Gamma\left(2-\gamma_{2}\right)}\right] \\
& \times \frac{\sum_{i=1}^{N}\left(\ln \left(\eta_{i} / a\right)\right)^{\beta-\delta_{1}}}{\Gamma\left(\beta-\delta_{1}+1\right)}+\frac{\left|\mu_{1}\right|}{\left|1-\mu_{1} \lambda_{1}\right|} \frac{(\ln (b / a))^{\beta}}{\Gamma(\beta+1)}, \\
& K_{3}=\frac{\left|\mu_{3}\right|}{|\Delta|}\left[\frac{\left|\mu_{1}\right|\left|\lambda_{1}\right|\left|\lambda_{2}\right|(\ln (b / a))^{2-\gamma_{1}}}{\Gamma\left(2-\gamma_{1}\right)\left|1-\mu_{1} \lambda_{1}\right|}\right. \\
& \left.+\frac{\left|\mu_{1}\right|\left|\mu_{2}\right|(\ln (b / a)) \sum_{i=1}^{N}\left(\ln \left(\eta_{i} / a\right)\right)^{1-\delta_{1}}}{\Gamma\left(2-\delta_{1}\right)\left|1-\mu_{1} \lambda_{1}\right|}+\frac{\left|\lambda_{2}\right|(\ln (b / a))^{2-\gamma_{1}}}{\Gamma\left(2-\gamma_{1}\right)}\right] \\
& \cdot \frac{\sum_{i=1}^{M}\left(\ln \left(\xi_{i} / a\right)\right)^{\alpha-\delta_{2}}}{\Gamma\left(\alpha-\delta_{2}+1\right)}+\frac{\left|\lambda_{2}\right|}{|\Delta|}\left[\frac{\left|\mu_{1}\right|\left|\mu_{3}\right|\left|\lambda_{1}\right|(\ln (b / a)) \sum_{i=1}^{M}\left(\ln \left(\xi_{i} / a\right)\right)^{1-\delta_{2}}}{\Gamma\left(2-\delta_{2}\right)\left|1-\mu_{1} \lambda_{1}\right|}\right. \\
& \left.+\frac{\left|\mu_{1}\right|\left|\lambda_{3}\right|(\ln (b / a))^{2-\gamma_{2}}}{\Gamma\left(2-\gamma_{2}\right)\left|1-\mu_{1} \lambda_{1}\right|}+\frac{\left|\mu_{3}\right|(\ln (b / a)) \sum_{i=1}^{M}\left(\ln \left(\xi_{i} / a\right)\right)^{1-\delta_{2}}}{\Gamma\left(2-\delta_{2}\right)}\right] \\
& \times \frac{(\ln (b / a))^{\alpha-\gamma_{1}}}{\Gamma\left(\alpha-\gamma_{1}+1\right)}+\frac{\left|\mu_{1}\right|}{\left|1-\lambda_{1} \mu_{1}\right|} \frac{(\ln (b / a))^{\alpha}}{\Gamma(\alpha+1)}, \\
& K_{4}=\frac{\left|\lambda_{3}\right|}{|\Delta|}\left[\frac{\left|\mu_{1}\right|\left|\lambda_{1}\right|\left|\lambda_{2}\right|(\ln (b / a))^{2-\gamma_{1}}}{\Gamma\left(2-\gamma_{1}\right)\left|1-\mu_{1} \lambda_{1}\right|}\right. \\
& \left.+\frac{\left|\mu_{1}\right|\left|\mu_{2}\right|(\ln (b / a)) \sum_{i=1}^{N}\left(\ln \left(\eta_{i} / a\right)\right)^{1-\delta_{1}}}{\Gamma\left(2-\delta_{1}\right)\left|1-\mu_{1} \lambda_{1}\right|}+\frac{\lambda_{2}(\ln (b / a))^{2-\gamma_{1}}}{\Gamma\left(2-\gamma_{1}\right)}\right] \\
& \cdot \frac{(\ln (b / a))^{\beta-\gamma_{2}}}{\Gamma\left(\beta-\gamma_{2}+1\right)}+\frac{\left|\mu_{2}\right|}{|\Delta|}\left[\frac{\left|\mu_{1}\right|\left|\mu_{3}\right|\left|\lambda_{1}\right|(\ln (b / a)) \sum_{i=1}^{M}\left(\ln \left(\xi_{i} / a\right)\right)^{1-\delta_{2}}}{\Gamma\left(2-\delta_{2}\right)\left|1-\mu_{1} \lambda_{1}\right|}\right. \\
& \left.+\frac{\left|\mu_{1}\right|\left|\lambda_{3}\right|(\ln (b / a))^{2-\gamma_{2}}}{\Gamma\left(2-\gamma_{2}\right)\left|1-\mu_{1} \lambda_{1}\right|}+\frac{\mu_{3}(\ln (b / a)) \sum_{i=1}^{M}\left(\ln \left(\xi_{i} / a\right)\right)^{1-\delta_{2}}}{\Gamma\left(2-\delta_{2}\right)}\right] \\
& \times \frac{\sum_{i=1}^{N}\left(\ln \left(\eta_{i} / a\right)\right)^{\beta-\delta_{1}}}{\Gamma\left(\beta-\delta_{1}+1\right)}+\left[\frac{\left|\lambda_{1}\right|\left|\mu_{1}\right|}{\left|1-\lambda_{1} \mu_{1}\right|}+1\right] \frac{(\ln (b / a))^{\beta}}{\Gamma(\beta+1)} \text {. }
\end{aligned}
$$

Now, we are in a position to present our main results. The methods used to prove the existence and uniqueness solutions of boundary value problem (3), (4) via Banach's contraction principle.

Theorem 8. Suppose that $f, g:[a, b] \times R \times R \rightarrow R$ are continuous functions. In addition, we assume that:

(H1) there exist constants $m_{i}$ and $n_{i}, i=1,2$, such that for all $t \in[a, b]$ and $u_{i}, v_{i} \in R, i=1,2$, we have

$$
\begin{gathered}
\left|f\left(t, u_{1}, v_{1}\right)-f\left(t, u_{2}, v_{2}\right)\right| \leq m_{1}\left|u_{1}-u_{2}\right|+m_{2}\left|v_{1}-v_{2}\right|, \\
\left|g\left(t, u_{1}, v_{1}\right)-g\left(t, u_{2}, v_{2}\right)\right| \leq n_{1}\left|u_{1}-u_{2}\right|+n_{2}\left|v_{1}-v_{2}\right| .
\end{gathered}
$$


Then, the system (3), (4) has a unique solution on $[a, b]$, if

$$
\left(K_{1}+K_{3}\right)\left(m_{1}+m_{2}\right)+\left(K_{2}+K_{4}\right)\left(n_{1}+n_{2}\right)<1
$$

Proof. Define $\sup _{t \in[a, b]} f(t, 0,0)=\sigma_{1}<\infty$ and $\sup _{t \in[a, b]} g(t, 0,0)=\sigma_{2}$ $<\infty$ and $r>0$ such that

$$
r>\frac{\left(K_{1}+K_{3}\right) \sigma_{1}+\left(K_{2}+K_{4}\right) \sigma_{2}}{1-\left[\left(K_{1}+K_{3}\right)\left(m_{1}+m_{2}\right)+\left(K_{2}+K_{4}\right)\left(n_{1}+n_{2}\right)\right]} .
$$

Now, we show that $\mathscr{T} B_{r} \subset B_{r}$, where $B_{r}=\{(u, v) \in X \times$ $Y:\|(u, v)\| \leq r\}$.

By assumption $(H 1)$, for $(u, v) \in B_{r}, t \in[a, b]$, we have that

$$
\begin{aligned}
|f(t, u(t), v(t))| & \leq|f(t, u(t), v(t))-f(t, 0,0)|+|f(t, 0,0)| \\
& \leq m_{1}|u(t)|+m_{2}|v(t)|+\sigma_{1} \leq m_{1}\|u\|+m_{2}\|v\|+\sigma_{1}
\end{aligned}
$$

$|g(t, u(t), v(t))| \leq n_{1}\|u\|+n_{2}\|v\|+\sigma_{2}$,

which leads to

$$
\begin{aligned}
\left|\mathscr{T}_{1}(u, v)(t)\right| \leq & \frac{\left|\mu_{3}\right|}{|\Delta|}\left[\frac{\left|\mu_{1}\left\|\mu_{2}\right\| \lambda_{1}\right|(\ln (b / a)) \sum_{i=1}^{N}\left(\ln \left(\eta_{i} / a\right)\right)^{1-\delta_{1}}}{\Gamma\left(2-\delta_{1}\right)\left|\left(1-\mu_{1} \lambda_{1}\right)\right|}\right. \\
& \left.+\frac{\left|\lambda_{1}\right|\left|\lambda_{2}\right|(\ln (b / a))^{2-\gamma_{1}}}{\Gamma\left(2-\gamma_{1}\right)\left|\left(1-\mu_{1} \lambda_{1}\right)\right|}+\frac{\left|\mu_{2}\right|(\ln (b / a)) \sum_{i=1}^{N}\left(\ln \left(\eta_{i} / a\right)\right)^{1-\delta_{1}}}{\Gamma\left(2-\delta_{1}\right)}\right] \\
& \times \frac{\sum_{i=1}^{M}\left(\ln \left(\xi_{i} / a\right)\right)^{\alpha-\delta_{2}}}{\Gamma\left(\alpha-\delta_{2}+1\right)}\left(m_{1}\|u\|+m_{2}\|v\|+\sigma_{1}\right) \\
& +\frac{\left|\lambda_{3}\right|}{|\Delta|}\left[\frac{\left|\mu_{1}\right|\left|\mu_{2}\right|\left|\lambda_{1}\right|(\ln (b / a)) \sum_{i=1}^{N}\left(\ln \left(\eta_{i} / a\right)\right)^{1-\delta_{1}}}{\Gamma\left(2-\delta_{1}\right)\left|\left(1-\mu_{1} \lambda_{1}\right)\right|}\right. \\
& \left.+\frac{\left|\lambda_{1}\right|\left|\lambda_{2}\right|(\ln (b / a))^{2-\gamma_{1}}}{\Gamma\left(2-\gamma_{1}\right)\left|\left(1-\mu_{1} \lambda_{1}\right)\right|}+\frac{\left|\mu_{2}\right|(\ln (b / a)) \sum_{i=1}^{N}\left(\ln \left(\eta_{i} / a\right)\right)^{1-\delta_{1}}}{\Gamma\left(2-\delta_{1}\right)}\right] \\
& \times \frac{(\ln (b / a))^{\beta-\gamma_{2}}}{\Gamma\left(\beta-\gamma_{2}+1\right)}\left(n_{1}\|u\|+n_{2}\|v\|+\sigma_{2}\right) \\
& +\frac{\left|\mu_{2}\right|}{|\Delta|}\left[\frac{\left|\mu_{1}\right|\left|\lambda_{1}\right|\left|\lambda_{3}\right|(\ln (b / a))^{2-\gamma_{2}}}{\Gamma\left(2-\gamma_{2}\right)\left|\left(1-\mu_{1} \lambda_{1}\right)\right|}\right. \\
& \left.+\frac{\left|\lambda_{1}\right|\left|\mu_{3}\right|(\ln (b / a)) \sum_{i=1}^{M}\left(\ln \left(\xi_{i} / a\right)\right)^{1-\delta_{2}}}{\Gamma\left(2-\delta_{2}\right)\left|\left(1-\mu_{1} \lambda_{1}\right)\right|}+\frac{\left|\lambda_{3}\right|(\ln (b / a))^{2-\gamma_{2}}}{\Gamma\left(2-\gamma_{2}\right)}\right] \\
& \times \frac{\sum_{i=1}^{N}\left(\ln \left(\eta_{i} / a\right)\right)^{\beta-\delta_{1}}}{\Gamma\left(\beta-\delta_{1}+1\right)}\left(n_{1}\|u\|+n_{2}\|v\|+\sigma_{2}\right) \\
& +\frac{\left|\lambda_{2}\right|}{|\Delta|}\left[\frac{\left|\mu_{1}\right|\left|\lambda_{1}\right|\left|\lambda_{3}\right|(\ln (b / a))^{2-\gamma_{2}}}{\Gamma\left(2-\gamma_{2}\right)\left|\left(1-\mu_{1} \lambda_{1}\right)\right|}+\frac{\left|\lambda_{1}\right|\left|\mu_{3}\right|(\ln (b / a)) \sum_{i=1}^{M}\left(\ln \left(\xi_{i} / a\right)\right)^{1-\delta_{2}}}{\Gamma\left(2-\delta_{2}\right)\left|\left(1-\mu_{1} \lambda_{1}\right)\right|}\right. \\
& \left.+\frac{\left.\left|\lambda_{3}\right|(\ln (b / a))^{2-\gamma_{2}}\right]}{\Gamma\left(2-\gamma_{2}\right)}\right] \times \frac{(\ln (b / a))^{\alpha-\gamma_{1}}}{\Gamma\left(\alpha-\gamma_{1}+1\right)}\left(m_{1}\|u\|+m_{2}\|v\|+\sigma_{1}\right) \\
& +\frac{\left|\lambda_{1}\right|}{\left|1-\mu_{1} \lambda_{1}\right|}\left[\left|\mu_{1}\right| \frac{(\ln (b / a))^{\alpha}}{\Gamma(\alpha+1)}\left(m_{1}\|u\|+m_{2}\|v\|+\sigma_{1}\right)\right. \\
& \left.+\frac{(\ln (b / a))^{\beta}}{\Gamma(\beta+1)}\left(n_{1}\|u\|+n_{2}\|v\|+\sigma_{2}\right)\right]+\frac{(\ln (b / a))^{\alpha}}{\Gamma(\alpha+1)}\left(m_{1}\|u\|+m_{2}\|v\|+\sigma_{1}\right) \\
= & K_{1}\left(m_{1}\|u\|+m_{2}\|v\|+\sigma_{1}\right)+K_{2}\left(n_{1}\|u\|+n_{2}\|v\|+\sigma_{2}\right) \\
= & \left.K_{1} m_{1}+K_{2} n_{1}\right)\|u\| \mid+\left(K_{1} m_{2}+K_{2} n_{2}\right)\|v\| \\
& +K_{1} \sigma_{1}+K_{2} \sigma_{2} \leq\left[K_{1}\left(m_{1}+m_{2}\right)+K_{2}\left(n_{1}+n_{2}\right)\right] r+K_{1} \sigma_{1}+k_{2} \sigma_{2} . \\
&
\end{aligned}
$$

Hence,

$\left\|\mathscr{T}_{1}(u, v)\right\| \leq\left[K_{1}\left(m_{1}+m_{2}\right)+K_{2}\left(n_{1}+n_{2}\right)\right] r+K_{1} \sigma_{1}+K_{2} \sigma_{2}$.

In the same way, we can obtain that

$$
\left\|\mathscr{T}_{2}(u, v)\right\| \leq\left[K_{3}\left(m_{1}+m_{2}\right)+K_{4}\left(n_{1}+n_{2}\right)\right] r+K_{3} \sigma_{1}+K_{4} \sigma_{2} .
$$

Consequently, it follows that

$$
\begin{aligned}
\|\mathscr{T}(u, v)\| \leq & {\left[K_{1}\left(m_{1}+m_{2}\right)+K_{2}\left(n_{1}+n_{2}\right)\right] r+K_{1} \sigma_{1}+K_{2} \sigma_{2} } \\
& +\left[K_{3}\left(m_{1}+m_{2}\right)+K_{4}\left(n_{1}+n_{2}\right)\right] r+K_{3} \sigma_{1}+K_{4} \sigma_{2} \leq r,
\end{aligned}
$$

which implies $\mathscr{T} B_{r} \subset B_{r}$. Next, we show that operator $\mathscr{T}$ is contraction mapping.

For any $\left(u_{1}, v_{1}\right),\left(u_{2}, v_{2}\right) \in X \times Y$ and for any $t \in[a, b]$, we obtain

$$
\begin{aligned}
& \left|\mathscr{T}_{1}\left(u_{1}, v_{1}\right)(t)-\mathscr{T}_{1}\left(u_{2}, v_{2}\right)(t)\right| \leq \frac{\left|\mu_{3}\right|}{|\Delta|} \\
& \cdot\left[\frac{\left|\mu_{1}\right|\left|\mu_{2}\right|\left|\lambda_{1}\right|(\ln (b / a)) \sum_{i=1}^{N}\left(\ln \left(\eta_{i} / a\right)\right)^{1-\delta_{1}}}{\Gamma\left(2-\delta_{1}\right)\left|\left(1-\mu_{1} \lambda_{1}\right)\right|}\right. \\
& +\frac{\left|\lambda_{1}\right|\left|\lambda_{2}\right|(\ln (b / a))^{2-\gamma_{1}}}{\Gamma\left(2-\gamma_{1}\right)\left|\left(1-\mu_{1} \lambda_{1}\right)\right|} \\
& \left.+\frac{\left|\mu_{2}\right|(\ln (b / a)) \sum_{i=1}^{N}\left(\ln \left(\eta_{i} / a\right)\right)^{1-\delta_{1}}}{\Gamma\left(2-\delta_{1}\right)}\right] \times \frac{\sum_{i=1}^{M}\left(\ln \left(\xi_{i} / a\right)\right)^{\alpha-\delta_{2}}}{\Gamma\left(\alpha-\delta_{2}+1\right)} \\
& \cdot\left(m_{1}\left\|u_{1}-u_{2}||+m_{2}\right\| v_{1}-v_{2} \mid\right)+\frac{\left|\lambda_{3}\right|}{|\Delta|} \\
& \cdot\left[\frac{\left|\mu_{1}\right|\left|\mu_{2}\right|\left|\lambda_{1}\right|(\ln (b / a)) \sum_{i=1}^{N}\left(\ln \left(\eta_{i} / a\right)\right)^{1-\delta_{1}}}{\Gamma\left(2-\delta_{1}\right)\left|\left(1-\mu_{1} \lambda_{1}\right)\right|}+\frac{\left|\lambda_{1}\right|\left|\lambda_{2}\right|(\ln (b / a))^{2-\gamma_{1}}}{\Gamma\left(2-\gamma_{1}\right)\left|\left(1-\mu_{1} \lambda_{1}\right)\right|}\right. \\
& \left.+\frac{\left|\mu_{2}\right|(\ln (b / a)) \sum_{i=1}^{N}\left(\ln \left(\eta_{i} / a\right)\right)^{1-\delta_{1}}}{\Gamma\left(2-\delta_{1}\right)}\right] \times \frac{(\ln (b / a))^{\beta-\gamma_{2}}}{\Gamma\left(\beta-\gamma_{2}+1\right)} \\
& \cdot\left(n_{1}\left\|u_{1}-u_{2}\right\|+n_{2}\left\|v_{1}-v_{2}\right\|\right)+\frac{\left|\mu_{2}\right|}{|\Delta|}\left[\frac{\left|\mu_{1}\right|\left|\lambda_{1}\right|\left|\lambda_{3}\right|(\ln (b / a))^{2-\gamma_{2}}}{\Gamma\left(2-\gamma_{2}\right)\left|\left(1-\mu_{1} \lambda_{1}\right)\right|}\right. \\
& \left.+\frac{\left|\lambda_{1}\right|\left|\mu_{3}\right|(\ln (b / a)) \sum_{i=1}^{M}\left(\ln \left(\xi_{i} / a\right)\right)^{1-\delta_{2}}}{\Gamma\left(2-\delta_{2}\right)\left|\left(1-\mu_{1} \lambda_{1}\right)\right|}+\frac{\left|\lambda_{3}\right|(\ln (b / a))^{2-\gamma_{2}}}{\Gamma\left(2-\gamma_{2}\right)}\right] \\
& \times \frac{\sum_{i=1}^{N}\left(\ln \left(\eta_{i} / a\right)\right)^{\beta-\delta_{1}}}{\Gamma\left(\beta-\delta_{1}+1\right)}\left(n_{1}\left\|u_{1}-u_{2}\right\|+n_{2}\left\|v_{1}-v_{2}\right\|\right)+\frac{\left|e_{2}\right|}{|\Delta|} \\
& \cdot\left[\frac{\left|\mu_{1}\right|\left|\lambda_{1}\right|\left|\lambda_{3}\right|(\ln (b / a))^{2-\gamma_{2}}}{\Gamma\left(2-\gamma_{2}\right)\left|\left(1-\mu_{1} \lambda_{1}\right)\right|}+\frac{\left|\lambda_{1}\right|\left|\mu_{3}\right|(\ln (b / a)) \sum_{i=1}^{M}\left(\ln \left(\xi_{i} / a\right)\right)^{1-\delta_{2}}}{\Gamma\left(2-\delta_{2}\right)\left|\left(1-\mu_{1} \lambda_{1}\right)\right|}\right. \\
& \left.+\frac{\left|\lambda_{3}\right|(\ln (b / a))^{2-\gamma_{2}}}{\Gamma\left(2-\gamma_{2}\right)}\right] \times \frac{(\ln (b / a))^{\alpha-\gamma_{1}}}{\Gamma\left(\alpha-\gamma_{1}+1\right)}\left(m_{1}\left\|u_{1}-u_{2}\right\|+m_{2}\left\|v_{1}-v_{2}\right\|\right) \\
& +\frac{\left|\lambda_{1}\right|}{\left|1-\mu_{1} \lambda_{1}\right|}\left[\left|\mu_{1}\right| \frac{(\ln (b / a))^{\alpha}}{\Gamma(\alpha+1)}\left(m_{1}\left\|u_{1}-u_{2}\right\|+m_{2}\left\|v_{1}-v_{2}\right\|\right)\right. \\
& \left.+\frac{(\ln (b / a))^{\beta}}{\Gamma(\beta+1)}\left(n_{1}\left\|u_{1}-u_{2}\right\|+n_{2}\left\|v_{1}-v_{2}\right\|\right)\right]+\frac{(\ln (b / a))^{\alpha}}{\Gamma(\alpha+1)} \\
& \cdot\left(m_{1}\left\|u_{1}-u_{2}\right\|+m_{2}\left\|v_{1}-v_{2}\right\|\right)=K_{1}\left(m_{1}\left\|u_{1}-u_{2}\right\|+m_{2}\left\|v_{1}-v_{2}\right\|\right) \\
& +K_{2}\left(n_{1}\left\|u_{1}-u_{2}\right\|+n_{2}\left\|v_{1}-v_{2}\right\|\right)=\left(K_{1} m_{1}+K_{2} n_{1}\right)\left\|u_{1}-u_{2}\right\| \\
& +\left(K_{1} m_{2}+K_{2} n_{2}\right)\left\|v_{1}-v_{2}\right\| \text {. }
\end{aligned}
$$


Therefore, we get the following inequality

$$
\begin{aligned}
& \left\|\mathscr{T}_{1}\left(u_{1}, v_{1}\right)(t)-\mathscr{T}_{1}\left(u_{2}, v_{2}\right)(t)\right\| \leq\left(K_{1}\left(m_{1}+m_{2}\right)\right. \\
& \left.\quad+K_{2}\left(n_{1}+n_{2}\right)\right)\left(\left\|u_{1}-u_{2}\right\|+\left\|v_{1}-v_{2}\right\|\right) .
\end{aligned}
$$

Similarly,

$$
\begin{aligned}
& \left\|\mathscr{T}_{2}\left(u_{1}, v_{1}\right)(t)-\mathscr{T}_{2}\left(u_{2}, v_{2}\right)(t)\right\| \leq\left(K_{3}\left(m_{1}+m_{2}\right)\right. \\
& \left.\quad+K_{4}\left(n_{1}+n_{2}\right)\right)\left(\left\|u_{1}-u_{2}\right\|+\left\|v_{1}-v_{2}\right\|\right) .
\end{aligned}
$$

From inequalities (43) and (44), it yields

$$
\begin{gathered}
\left\|\mathscr{T}\left(u_{1}, v_{1}\right)(t)-\mathscr{T}\left(u_{2}, v_{2}\right)\right\| \leq\left[\left(K_{1}+K_{3}\right)\left(m_{1}+m_{2}\right)\right. \\
\left.+\left(K_{2}+K_{4}\right)\left(n_{1}+n_{2}\right)\right]\left(\left\|u_{1}-u_{2}\right\|+\left\|v_{1}-v_{2}\right\|\right) .
\end{gathered}
$$

Since $\quad\left(K_{1}+K_{3}\right)\left(m_{1}+m_{2}\right)+\left(K_{2}+K_{4}\right)\left(n_{1}+n_{2}\right)<1$, therefore, $\mathscr{T}$ is a contraction operator. So, by applying Banach's fixed point theorem, the operator $\mathscr{T}$ has a unique fixed point in $B_{r}$. Hence, there exists a unique solution of problem (3), (4) on $[a, b]$.

Now, we prove our second existence result via the LeraySchauder alternative.

Lemma 9 (Leray-Schauder alternative [39]). Let $F: E \rightarrow E$ be a completely continuous operator (i.e., a map restricted to any bounded set in $E$ is compact). Let

$$
\varepsilon(F)=\{x \in E: x=\lambda F(x) \text { forsome } 0<\lambda<1\} .
$$

Then, either the set $\varepsilon(F)$ is unbounded or $F$ has at least one fixed point.

Theorem 10. Assume that:

(H2) $f, g:[a, b] \times R \times R \rightarrow R$ are continuous functions and there exist real constants $a_{i}, b_{i} \geq 0(i=0,1,2)$ and $a_{0}>0$, $b_{0}>0$ such that $\forall x_{i} \in R(i=1,2$,$) we have$

$$
\begin{aligned}
& \left|f\left(t, x_{1}, x_{2}\right)\right| \leq a_{0}+a_{1}\left|x_{1}\right|+a_{2}\left|x_{2}\right|, \\
& \left|g\left(t, x_{1}, x_{2}\right)\right| \leq b_{0}+b_{1}\left|x_{1}\right|+b_{2}\left|x_{2}\right| .
\end{aligned}
$$

If $\left(K_{1}+K_{3}\right) a_{1}+\left(K_{2}+K_{4}\right) b_{1}<1$ and $\left(K_{1}+K_{3}\right) a_{2}+($ $\left.K_{2}+K_{4}\right) b_{2}<1$ then system (3), (4) has at least one solution on $[a, b]$.

Proof. By the continuity of functions $f, g$ on $[a, b] \times R \times R$, the operator $\mathscr{T}$ is continuous. Now, we show that the operator $\mathscr{T}: X \times Y \rightarrow X \times Y$ is completely continuous. Let $\Omega \subset X \times$ $Y$ be bounded. Then, there exist two positive constants, $M_{1}$ and $M_{2}$, such that

$$
|f(t, u(t), v(t))| \leq M_{1},|g(t, u(t), v(t))| \leq M_{2} \forall(u, v) \in \Omega .
$$

Then, for any $(u, v) \in \Omega$, we have

$$
\begin{aligned}
& \left|\mathscr{T}_{1}(u, v)(t)\right| \leq \frac{\left|\mu_{3}\right|}{|\Delta|}\left[\frac{\left|\mu_{1}\right|\left|\mu_{2}\right|\left|\lambda_{1}\right|(\ln (b / a)) \sum_{i=1}^{N}\left(\ln \left(\eta_{i} / a\right)\right)^{1-\delta_{1}}}{\Gamma\left(2-\delta_{1}\right)\left|1-\mu_{1} \lambda_{1}\right|}\right. \\
& +\frac{\left|\lambda_{1}\right|\left|\lambda_{2}\right|(\ln (b / a))^{2-\gamma_{1}}}{\Gamma\left(2-\gamma_{1}\right)\left|1-\mu_{1} \lambda_{1}\right|} \\
& \left.+\frac{\left|\mu_{2}\right|(\ln (b / a)) \sum_{i=1}^{N}\left(\ln \left(\eta_{i} / a\right)\right)^{1-\delta_{1}}}{\Gamma\left(2-\delta_{1}\right)}\right] \\
& \times \frac{\sum_{i=1}^{M}\left(\ln \left(\xi_{i} / a\right)\right)^{\alpha-\delta_{2}}}{\Gamma\left(\alpha-\delta_{2}+1\right)} M_{1}+\frac{\left|\lambda_{3}\right|}{|\Delta|} \\
& \cdot\left[\frac{\left|\mu_{1}\right|\left|\mu_{2}\right|\left|\lambda_{1}\right|(\ln (b / a)) \sum_{i=1}^{N}\left(\ln \left(\eta_{i} / a\right)\right)^{1-\delta_{1}}}{\Gamma\left(2-\delta_{1}\right)\left|1-\mu_{1} \lambda_{1}\right|}\right. \\
& +\frac{\left|\lambda_{1}\right|\left|\lambda_{2}\right|(\ln (b / a))^{2-\gamma_{1}}}{\Gamma\left(2-\gamma_{1}\right)\left|1-\mu_{1} \lambda_{1}\right|} \\
& \left.+\frac{\left|\mu_{2}\right|(\ln (b / a)) \sum_{i=1}^{N}\left(\ln \left(\eta_{i} / a\right)\right)^{1-\delta_{1}}}{\Gamma\left(2-\delta_{1}\right)}\right] \\
& \times \frac{(\ln (b / a))^{\beta-\gamma_{2}}}{\Gamma\left(\beta-\gamma_{2}+1\right)} M_{2}+\frac{\left|\mu_{2}\right|}{|\Delta|}\left[\frac{\left|\mu_{1}\right|\left|\lambda_{1}\right| \lambda_{3} \mid(\ln (b / a))^{2-\gamma_{2}}}{\Gamma\left(2-\gamma_{2}\right)\left|1-\mu_{1} \lambda_{1}\right|}\right. \\
& +\frac{\left|\lambda_{1}\right|\left|\mu_{3}\right|(\ln (b / a)) \sum_{i=1}^{M}\left(\ln \left(\xi_{i} / a\right)\right)^{1-\delta_{2}}}{\Gamma\left(2-\delta_{2}\right)\left|1-\mu_{1} \lambda_{1}\right|} \\
& \left.+\frac{\left|\lambda_{3}\right|(\ln (b / a))^{2-\gamma_{2}}}{\Gamma\left(2-\gamma_{2}\right)}\right] \times \frac{\sum_{i=1}^{N}\left(\ln \left(\eta_{i} / a\right)\right)^{\beta-\delta_{1}}}{\Gamma\left(\beta-\delta_{1}+1\right)} M_{2} \\
& +\frac{\left|\lambda_{2}\right|}{|\Delta|}\left[\frac{\left|\mu_{1}\right|\left|\lambda_{1}\right|\left|\lambda_{3}\right|(\ln (b / a))^{2-\gamma_{2}}}{\Gamma\left(2-\gamma_{2}\right)\left|1-\mu_{1} \lambda_{1}\right|}\right. \\
& +\frac{\left|\lambda_{1}\right|\left|\mu_{3}\right|(\ln (b / a)) \sum_{i=1}^{M}\left(\ln \left(\xi_{i} / a\right)\right)^{1-\delta_{2}}}{\Gamma\left(2-\delta_{2}\right)\left|1-\mu_{1} \lambda_{1}\right|} \\
& \left.+\frac{\left|\lambda_{3}\right|(\ln (b / a))^{2-\gamma_{2}}}{\Gamma\left(2-\gamma_{2}\right)}\right] \times \frac{(\ln (b / a))^{\alpha-\gamma_{1}}}{\Gamma\left(\alpha-\gamma_{1}+1\right)} M_{1} \\
& +\frac{\left|\lambda_{1}\right|}{\left|1-\mu_{1} \lambda_{1}\right|}\left[\left|\mu_{1}\right| \frac{(\ln (b / a))^{\alpha}}{\Gamma(\alpha+1)} M_{1}+\frac{(\ln (b / a))^{\beta}}{\Gamma(\beta+1)} M_{2}\right] \\
& +\frac{(\ln (b / a))^{\alpha}}{\Gamma(\alpha+1)} M_{1} \text {, }
\end{aligned}
$$

which yields,

$$
\left\|\mathscr{T}_{1}(u, v)\right\| \leq K_{1} M_{1}+K_{2} M_{2} .
$$

In the same way, we can obtain that $\left\|\mathscr{T}_{2}(u, v)\right\| \leq K_{3} M_{1}$ $+K_{4} M_{2}$. Hence, from the above inequalities, we get that the operator $\mathscr{T}$ is uniformly bounded, since $\|\mathscr{T}(u, v)\| \leq\left(K_{1}\right.$ $\left.+K_{3}\right) M_{1}+\left(K_{2}+K_{4}\right) M_{2}$.

Next, we show that $\mathscr{T}$ is equicontinuous. For any $(u, v)$ $\in \Omega$, and $\tau_{1}, \tau_{2} \in[a, b]$ with $\tau_{1}<\tau_{2}$. Then, we have

$$
\begin{aligned}
& \left|\mathscr{T}_{1}(u, v)\left(\tau_{2}\right)-\mathscr{T}_{1}(u, v)\left(\tau_{1}\right)\right| \leq \frac{M_{1}\left|\mu_{2}\right|\left|\mu_{3}\right| \sum_{i=1}^{N}\left(\ln \left(\eta_{i} / a\right)\right)^{1-\delta_{1}}}{|\Delta| \Gamma\left(2-\delta_{1}\right)} \\
& \quad \frac{\sum_{i=1}^{M}\left(\ln \left(\xi_{i} / a\right)\right)^{\alpha-\delta_{2}}}{\Gamma\left(\alpha-\delta_{2}+1\right)}\left|\left(\ln \frac{\tau_{2}}{a}\right)-\left(\ln \frac{\tau_{1}}{a}\right)\right|
\end{aligned}
$$




$$
\begin{aligned}
& +\frac{M_{2}\left|\mu_{2}\right|\left|\lambda_{3}\right| \sum_{i=1}^{N}\left(\ln \left(\eta_{i} / a\right)\right)^{1-\delta_{1}}}{|\Delta| \Gamma\left(2-\delta_{1}\right)} \frac{(\ln (b / a))^{\beta-\gamma_{2}}}{\Gamma\left(\beta-\gamma_{2}+1\right)} \mid\left(\ln \frac{\tau_{2}}{a}\right) \\
& -\left(\ln \frac{\tau_{1}}{a}\right) \mid+\frac{M_{2}|| \mu_{2}\left|\lambda_{3}\right|(\ln (b / a))^{1-\gamma_{2}}}{|\Delta| \Gamma\left(2-\gamma_{2}\right)} \frac{\sum_{i=1}^{N}\left(\ln \left(\eta_{i} / a\right)\right)^{\beta-\delta_{1}}}{\Gamma\left(\beta-\delta_{1}+1\right)} \\
& \cdot\left|\left(\ln \frac{\tau_{2}}{a}\right)-\left(\ln \frac{\tau_{1}}{a}\right)\right|+M_{1} \frac{\left|\lambda_{2}\right|\left|\lambda_{3}\right|(\ln (b / a))^{1-\gamma_{2}}}{|\Delta| \Gamma\left(2-\gamma_{2}\right)} \frac{(\ln (b / a))^{\alpha-\gamma_{1}}}{\Gamma\left(\alpha-\gamma_{1}+1\right)} \\
& \cdot\left|\left(\ln \frac{\tau_{2}}{a}\right)-\left(\ln \frac{\tau_{1}}{a}\right)\right|+M_{1} \mid \frac{1}{\Gamma(\alpha)} \int_{a}^{\tau_{2}}\left(\ln \frac{\tau_{2}}{s}\right)^{\alpha-1} \\
& \cdot \frac{d s}{s}-\frac{1}{\Gamma(\alpha)} \int_{a}^{\tau_{1}}\left(\ln \frac{\tau_{1}}{s}\right)^{\alpha-1} \frac{d s}{s} \mid \leq \frac{M_{1}\left|\mu_{2}\right|\left|\mu_{3}\right| \sum_{i=1}^{N}\left(\ln \left(\eta_{i} / a\right)\right)^{1-\delta_{1}}}{|\Delta| \Gamma\left(2-\delta_{1}\right)} \\
& \frac{\sum_{i=1}^{M}\left(\ln \left(\xi_{i} / a\right)\right)^{\alpha-\delta_{2}}}{\Gamma\left(\alpha-\delta_{2}+1\right)}\left|\left(\ln \frac{\tau_{2}}{a}\right)-\left(\ln \frac{\tau_{1}}{a}\right)\right| \\
& +\frac{M_{2}\left|\mu_{2}\right|\left|\mu_{3}\right| \sum_{i=1}^{N}\left(\ln \left(\eta_{i} / a\right)\right)^{1-\delta_{1}}}{|\Delta| \Gamma\left(2-\delta_{1}\right)} \frac{(\ln (b / a))^{\beta-\gamma_{2}}}{\Gamma\left(\beta-\gamma_{2}+1\right)} \mid\left(\ln \frac{\tau_{2}}{a}\right) \\
& -\left(\ln \frac{\tau_{1}}{a}\right) \mid+\frac{M_{2}|| \mu_{2}\left|\lambda_{3}\right|(\ln (b / a))^{1-\gamma_{2}}}{|\Delta| \Gamma\left(2-\gamma_{2}\right)} \frac{\sum_{i=1}^{N}\left(\ln \left(\eta_{i} / a\right)\right)^{\beta-\delta_{1}}}{\Gamma\left(\beta-\delta_{1}+1\right)} \\
& \cdot\left|\left(\ln \frac{\tau_{2}}{a}\right)-\left(\ln \frac{\tau_{1}}{a}\right)\right|+M_{1} \frac{\left|\lambda_{2}\right|\left|\lambda_{3}\right|(\ln (b / a))^{1-\gamma_{2}}}{|\Delta| \Gamma\left(2-\gamma_{2}\right)} \frac{(\ln (b / a))^{\alpha-\gamma_{1}}}{\Gamma\left(\alpha-\gamma_{1}+1\right)} \\
& \cdot\left|\left(\ln \frac{\tau_{2}}{a}\right)-\left(\ln \frac{\tau_{1}}{a}\right)\right|+M_{1}\left|\frac{1}{\Gamma(\alpha)} \int_{a}^{\tau_{1}}\left[\left(\ln \frac{\tau_{2}}{s}\right)^{\alpha-1}-\left(\ln \frac{\tau_{1}}{s}\right)^{\alpha-1}\right] \frac{d s}{s}\right| \\
& +M_{1}\left|\frac{1}{\Gamma(\alpha)} \int_{\tau_{1}}^{\tau_{2}}\left(\ln \frac{\tau_{2}}{s}\right)^{\alpha-1} \frac{d s}{s}\right|=\frac{M_{1}\left|\mu_{2}\right|\left|\mu_{3}\right| \sum_{i=1}^{N}\left(\ln \left(\eta_{i} / a\right)\right)^{1-\delta_{1}}}{|\Delta| \Gamma\left(2-\delta_{1}\right)} \\
& \frac{\sum_{i=1}^{M}\left(\ln \left(\xi_{i} / a\right)\right)^{\alpha-\delta_{2}}}{\Gamma\left(\alpha-\delta_{2}+1\right)}\left|\left(\ln \frac{\tau_{2}}{a}\right)-\left(\ln \frac{\tau_{1}}{a}\right)\right| \\
& +\frac{M_{2}\left|\mu_{2}\right|\left|\lambda_{3}\right| \sum_{i=1}^{N}\left(\ln \left(\eta_{i} / a\right)\right)^{1-\delta_{1}}}{|\Delta| \Gamma\left(2-\delta_{1}\right)} \frac{(\ln (b / a))^{\beta-\gamma_{2}}}{\Gamma\left(\beta-\gamma_{2}+1\right)} \mid\left(\ln \frac{\tau_{2}}{a}\right) \\
& -\left(\ln \frac{\tau_{1}}{a}\right) \mid+\frac{M_{2}|| \mu_{2}\left|\lambda_{3}\right|(\ln (b / a))^{1-\gamma_{2}}}{|\Delta| \Gamma\left(2-\gamma_{2}\right)} \frac{\sum_{i=1}^{N}\left(\ln \left(\eta_{i} / a\right)\right)^{\beta-\delta_{1}}}{\Gamma\left(\beta-\delta_{1}+1\right)} \\
& \cdot\left|\left(\ln \frac{\tau_{2}}{a}\right)-\left(\ln \frac{\tau_{1}}{a}\right)\right|+M_{1} \frac{\left|\lambda_{2}\right|\left|\lambda_{3}\right|(\ln (b / a))^{1-\gamma_{2}}}{|\Delta| \Gamma\left(2-\gamma_{2}\right)} \frac{(\ln (b / a))^{\alpha-\gamma_{1}}}{\Gamma\left(\alpha-\gamma_{1}+1\right)} \\
& \cdot\left|\left(\ln \frac{\tau_{2}}{a}\right)-\left(\ln \frac{\tau_{1}}{a}\right)\right|+\frac{M_{1}}{\Gamma(\alpha+1)}\left[2\left(\ln \frac{\tau_{2}}{\tau_{1}}\right)^{\alpha}+\left|\left(\ln \frac{\tau_{2}}{a}\right)^{\alpha}-\left(\ln \frac{\tau_{1}}{a}\right)^{\alpha}\right|\right] .
\end{aligned}
$$

Therefore, we obtain

$$
\left|\mathscr{T}_{1}(u, v)\left(\tau_{2}\right)-\mathscr{T}_{1}(u, v)\left(\tau_{1}\right)\right| \longrightarrow 0, a s \tau_{1} \longrightarrow \tau_{2} .
$$

Analogously, we can get the following inequality:

$$
\left|\mathscr{T}_{2}(u, v)\left(\tau_{2}\right)-\mathscr{T}_{2}(u, v)\left(\tau_{1}\right)\right| \longrightarrow 0, a s \tau_{1} \longrightarrow \tau_{2} .
$$

Then we can easily show that the operator $\mathscr{T}(u, v)$ is equicontinuous. As a consequence of steps together with the Arzel $a^{\prime}$-Ascoli theorem, we get that the operator $\mathscr{T}(u, v$ ) is completely continuous.

Finally, it will be verified that the set $\varepsilon=\{(u, v) \in X \times Y$ $:(u, v)=\lambda \mathscr{T}(u, v), 0 \leq \lambda \leq 1\}$ is bounded. Let $(u, v) \in \mathcal{E}$, with $(u, v)=\lambda \mathscr{T}(u, v)$. For any $t \in[a, b]$, we have

$$
u(t)=\lambda \mathscr{T}_{1}(u, v)(t), v(t)=\lambda \mathscr{T}_{2}(u, v)(t) .
$$

Then, we have

$$
\begin{aligned}
\|u(t)\| & \leq K_{1}\left(a_{0}+a_{1}\|u\|+a_{2}\|v\|\right)+K_{2}\left(b_{0}+b_{1}\|u\|+b_{2}\|v\|\right) \\
& =K_{1} a_{0}+K_{2} b_{0}+\left(K_{1} a_{1}+K_{2} b_{1}\right)\|u\|+\left(K_{1} a_{2}+K_{2} b_{2}\right)\|v\|, \\
\|v(t)\| & \leq K_{3}\left(a_{0}+a_{1}\|u\|+a_{2}\|v\|\right)+K_{4}\left(b_{0}+b_{1}\|u\|+b_{2}\|v\|\right) \\
& =K_{3} a_{0}+K_{4} b_{0}+\left(K_{3} a_{1}+K_{4} b_{1}\right)\|u\|+\left(K_{3} a_{2}+K_{4} b_{2}\right)\|v\|,
\end{aligned}
$$

which implies that

$$
\begin{aligned}
\|u\| & +\|v\| \leq\left(K_{1}+K_{3}\right) a_{0}+\left(K_{2}+K_{4}\right) b_{0} \\
& +\left[\left(K_{1}+K_{3}\right) a_{1}+\left(K_{2}+K_{4}\right) b_{1}\right]\|u\| \\
& +\left[\left(K_{1}+K_{3}\right) a_{2}+\left(K_{2}+K_{4}\right) b_{2}\right]\|v\| .
\end{aligned}
$$

Consequently,

$$
\|(u, v)\| \leq \frac{\left(K_{1}+K_{3}\right) a_{0}+\left(K_{2}+K_{4}\right) b_{0}}{K_{0}},
$$

where

$$
\begin{aligned}
K_{0}= & \min \left\{1-\left[\left(K_{1}+K_{3}\right) a_{1}+\left(K_{2}+K_{4}\right) b_{1}\right], 1\right. \\
& \left.-\left[\left(K_{1}+K_{3}\right) a_{2}+\left(K_{2}+K_{4}\right) b_{2}\right]\right\},
\end{aligned}
$$

which proves that $\varepsilon$ is bounded. Therefore, by applying Lemma 9, the operator $\mathscr{T}$ has at least one fixed point in $\Omega$. Therefore, we deduce that the boundary value problem (3), (4) has at least one solution on $[a, b]$.

\section{Some Examples}

In this section, we give an example to illustrate our main results.

Example 11. Consider the following system of CaputoHadamard boundary value problem:

$$
\left\{\begin{array}{l}
{ }^{c} \mathscr{D}_{1^{+}}^{3 / 2} u(t)=f(t, u(t), v(t)), t \in[1, e], \\
{ }^{c} \mathscr{D}_{1^{+}}^{3 / 2} v(t)=g f(t, u(t), v(t)), t \in[1, e], \\
u(1)=v(e), 1 / 2^{c} \mathscr{D}_{1^{+}}^{1 / 2} u(e)=1 / 3^{c} \mathscr{D}_{1^{+}}^{1 / 3} v(3 / 2)+1 / 3^{c} \mathscr{D}_{1^{+}}^{1 / 3} v(4 / 3), \\
v(1)=2 u(e), 1 / 4^{c} \mathscr{D}_{1^{+}}^{1 / 4} v(e)=1 / 5^{c} \mathscr{D}_{1^{+}}^{1 / 5} u(5 / 3)+1 / 5^{c} \mathscr{D}_{1^{+}}^{1 / 5} u(5 / 4) .
\end{array}\right.
$$

Here, $\alpha=\beta=3 / 2, a=1, b=e, \gamma_{1}=1 / 2, \gamma_{2}=1 / 4, \delta_{1}=1 / 3$, $\delta_{2}=1 / 5, N=M=2, \eta_{1}=3 / 2, \eta_{2}=4 / 3, \xi_{1}=5 / 3, \xi_{2}=5 / 4, \lambda_{1}=$ $1, \lambda_{2}=1 / 2, \lambda_{3}=1 / 4, \mu_{1}=2, \mu_{2}=1 / 3, \mu_{3}=1 / 5$. By simple calculation, we found that $\Delta=0.078172, K_{1}=10.36402, K_{2}=$ $8.38734, K_{3}=11.58173, K_{4}=11.18721$.

(i) Let two nonlinear functions $f, g:[1, e] \times R \times R \longrightarrow R$ be given by 


$$
\begin{aligned}
& f(t, x, y)=\frac{1}{15 \sqrt{24+t^{2}}} \frac{|x|}{1+|x(t)|}+\frac{\sin y(t)}{\left(64+t^{2}\right)}+\frac{1}{2}, \\
& g(t, x, y)=\frac{\sin (|x|)}{124+t^{2}}+\frac{\tan ^{-1}(y)}{120 t^{2}+2}+\frac{2}{3} .
\end{aligned}
$$

Note that

$$
\begin{aligned}
\left|f\left(t, x_{1}, x_{2}\right)-f\left(t, y_{1}, y_{2}\right)\right| & \leq \frac{1}{75}\left|x_{1}-x_{2}\right|+\frac{1}{65}\left|y_{1}-y_{2}\right|, \\
\left|g\left(t, x_{1}, x_{2}\right)-g\left(t, y_{1}, y_{2}\right)\right| & \leq \frac{1}{125}\left|x_{1}-x_{2}\right|+\frac{1}{122}\left|y_{1}-y_{2}\right|,
\end{aligned}
$$

we obtain $\left(K_{1}+K_{3}\right)(1 / 75+1 / 65)+\left(K_{2}+K_{4}\right)(1 / 125+1 /$ $122) \approx 0.9552435311<1$. Thus, all the conditions of Theorem 8 are satisfied. Problem (59) with (60) and (61)has a unique solution on $[1, e]$.

(ii) Let two nonlinear functions $f, g:[1, e] \times R \times R \rightarrow R$ be given by

$$
\begin{aligned}
& f(t, x, y)=\frac{e^{-2 t}}{4}+\frac{x^{2} \cos ^{2} t}{39(1+|x|)}+\frac{|y|^{4} \sin ^{2} t}{45\left(1+y^{3}\right)}, \\
& g(t, x, y)=\frac{2}{t^{2}+2}+\frac{\sin x}{12(t+4)}+\frac{\tan ^{-1} y}{14\left(3+t^{2}\right)} .
\end{aligned}
$$

Note that

$$
\begin{aligned}
& |f(t, x, y)| \leq \frac{1}{4}+\frac{1}{39}|x|+\frac{1}{45}|y|, \\
& |g(t, x, y)| \leq \frac{2}{3}+\frac{1}{60}|x|+\frac{1}{56}|y| .
\end{aligned}
$$

We get $a_{1}=1 / 39, a_{2}=1 / 45, b_{1}=1 / 60, b_{2}=1 / 56$. By simple calculation, we have $\left(K_{1}+K_{3}\right) a_{1}+\left(K_{2}+K_{4}\right) b_{1} \approx$ $0.8960930769<1$ and $\left(K_{1}+K_{3}\right) a_{2}+\left(K_{2}+K_{4}\right) b_{2} \approx$ $0.8434206667<1$. By Theorem 10 , the coupled boundary value problem (59) with (63) and (64) has at least one positive solution on $[1, \mathrm{e}]$.

\section{Conclusions}

In this paper, we studied existence and uniqueness of solutions for the system of Caputo-Hadamard fractional differential equations with multipoint boundary conditions. The existence theory of solutions of a Caputo-Hadamard system using a variety of fixed point theorems. The Leray-Schauder alternative was applied to prove existence, while the uniqueness result was obtained via the Banach contradiction mapping principle. Finally, we have given two examples to demonstrate our result.

\section{Data Availability}

No data were used to support this study.

\section{Conflicts of Interest}

There is no competing interest among the authors regarding the publication of the article.

\section{Authors' Contributions}

All authors contributed equally and significantly in writing this article. All authors read and approved the final manuscript.

\section{References}

[1] O. P. Agrawal, "A general solution for a fourth-order fractional diffusion-wave equation defined in a bounded domain," Computers and Structures, vol. 79, pp. 1497-1501, 2001.

[2] O.P. Agrawal, "Analytical schemes for a new class of fractional differential equations," Journal of Physics A: Mathematical and Theoretical, vol. 40, pp. 5469-5477, 2007.

[3] O. P. Agrawal, "Solution for a fractional diffusion-wave equation defined in a bounded domain," Nonlinear Dynamics, vol. 29, pp. 145-155, 2002.

[4] R. P. Agarwal, M. Benchohra, and S. Hamani, "Boundary value problems for fractional differential equations," Georgian Mathematical Journal, vol. 16, pp. 401-411, 2009.

[5] J. Hadamard, "Essai surletude des fonctions donnees par leur developpment de Taylor," Journal de Mathématiques Pures et Appliquées, vol. 8, pp. 101-186, 1892.

[6] A. A. Kilbas, "Hadamard-type fractional calculus," Journal of the Korean Mathematical Society, vol. 38, pp. 1191-1204, 2001.

[7] A. A. Kilbas, H. M. Srivastava, and J. J. Trujillo, Theory and Applications of Fractional Differential Equations, Elsevier, Amsterdam, 2006.

[8] I. Podlubny, Fractional Differential Equations, Academic Press, New York, 1999.

[9] K. S. Miller and B. Ross, An Introduction to the Fractional Calculus and Fractional Differential Equations, Wiley, New York, 1993.

[10] S. G. Samko, A. A. Kilbas, and O. I. Marichev, Fractional Integrals and Derivatives, Theory and Applications, Gordon and Breach, Yverdon, Switzerland, 1993.

[11] K. Diethelm, The Analysis of Fractional Differential Equations, Springer, Berlin, 2010.

[12] Y. Zhou, Basic Theory of Fractional Differential Equations, World Scientific, Singapore, 2014.

[13] Z. M. Ge and C. Y. Ou, "Chaos synchronization of fractional order modified Duffing systems with parameters excited by a chaotic signal," Chaos, Solitons and Fractals, vol. 35, pp. 705717, 2008.

[14] M. Faieghi, S. Kuntanapreeda, H. Delavari, and D. Baleanu, "LMI-based stabilization of a class of fractional-order chaotic systems,” Nonlinear Dynamics, vol. 72, pp. 301-309, 2013.

[15] F. Zhang, G. Chen, C. Li, and J. Kurths, "Chaos synchronization in fractional differential systems," Philosophical Transactions of the Royal Society A: Mathematical, Physical and Engineering Sciences, vol. 371, article 20120155, 2013.

[16] I. M. Sokolov, J. Klafter, and A. Blumen, "Fractional kinetics," Physics Today, vol. 55, pp. 48-54, 2002.

[17] R. Metzler and J. Klafter, "The random walk's guide to anomalous diffusion: a fractional dynamics approach," Physics Reports, vol. 339, pp. 1-77, 2000. 
[18] I. Petras and R. L. Magin, "Simulation of drug uptake in a two compartmental fractional model for a biological system," Communications in Nonlinear Science and Numerical Simulation, vol. 16, pp. 4588-4595, 2011.

[19] Y. Ding, Z. Wang, and H. Ye, "Optimal control of a fractionalorder HIV-immune system with memory," IEEE Transactions on Control Systems Technology, vol. 20, pp. 763-769, 2012.

[20] M. Javidi and B. Ahmad, "Dynamic analysis of time fractional order phytoplankton-toxic phytoplankton-zooplankton system," Ecological Modelling, vol. 318, pp. 8-18, 2015.

[21] I. Grigorenko and E. Grigorenko, "Chaotic dynamics of the fractional Lorenz system," Physical Review Letters, vol. 91, article 034101, 2003.

[22] M. Ostoja-Starzewski, "Towards thermoelasticity of fractal media," Journal of Thermal Stresses, vol. 30, pp. 889-896, 2007.

[23] Y. Z. Povstenko, Fractional Thermoelasticity, Springer, New York, NY, USA, 2015.

[24] A. Alsaedi, S. Aljoudi, and B. Ahmad, "Existence of solutions for Riemann-Liouville type coupled systems of fractional integro-differential equations and boundary conditions," Electronic Journal of Differential Equations, vol. 2016, p. 211, 2016.

[25] B. Ahmad, S. K. Ntouyas, and A. Alsaedi, "On a coupled system of fractional differential equations with coupled nonlocal and integral boundary conditions," Chaos, Solitons and Fractals, vol. 83, pp. 234-241, 2016.

[26] B. Ahmad and R. Luca, "Existence of solutions for a system of fractional differential equations with coupled nonlocal boundary conditions," Fractional Calculus and Applied Analysis, vol. 21, no. 2, pp. 423-441, 2018.

[27] S. N. Rao, "Existence and uniqueness of solutions for a nonlinear coupled system of fractional differential equationson time scales," Differential Equations and Dynamical Systems, 2018.

[28] B. Ahmad, S. K. Ntouyas, and A. Alsaedi, "Fractional order differential systems involving right Caputo and left RiemannLiouville fractional derivatives with nonlocal coupled conditions," Boundary Value Problems, vol. 2019, no. 109, 2019.

[29] S. N. Rao and M. Alesemi, "On a coupled system of fractional differential equations with nonlocal non-separated boundary conditions," Advances in Difference Equations, vol. 2019, Article ID 97, 2019.

[30] Y. Arioua and N. Benhamidouche, "Boundary value problem for Caputo-Hadamard fractional differential equations," Surveys in Mathematics and its Applications, vol. 12, pp. 103115, 2017.

[31] S. Asawasamrit, S. K. Ntouyas, J. Tariboon, and W. Nithiarayaphaks, "Coupled systems of sequential Caputo and Hadamard fractional differential equations with coupled separated boundary conditions," Symmetry, vol. 10, p. 701, 2018.

[32] B. Ahmad and S. K. Ntouyas, "A fully Hadamard type integral boundary value problem of a coupled system of fractional differential equations," Fractional Calculus and Applied Analysis, vol. 17, pp. 348-360, 2014.

[33] B. Ahmad and S. K. Ntouyas, "On Hadamard fractional integro-differential boundary value problems," Journal of Applied Mathematics and Computing, vol. 47, pp. 119-131, 2015.

[34] S. Aljoudi, B. Ahmad, and A. Alsaedi, "Existence and uniqueness results for a coupled system of Caputo-Hadamard fractional differential equations with nonlocal Hadamard type integral boundary conditions," Fractal and Fractional, vol. 4, p. 13, 2020, 2020.

[35] B. Ahmad and J. J. Nieto, "Existence results for a coupled system of nonlinear fractional differential equations with threepoint boundary conditions," Computers \& Mathematcs with Applications, vol. 58, pp. 1838-1843, 2009.

[36] H. H. Alsulami, S. K. Ntouyas, R. P. Agarwal, B. Ahmad, and A. Alsaedi, "A study of fractional-order coupled systems with a new concept of coupled non-separated boundary conditions," Boundary Value Problems, vol. 2017, Article ID 68, 2017.

[37] S. N. Rao, "The nonexistence of positive solutions for a coupled system of non-separated boundary value problems," Differential Equations and Dynamical Systems, 2019.

[38] F. Jarad, D. Baleanu, and A. Abdeljawad, "Caputo-type modification of the Hadamard fractional derivatives," Advances in Difference Equations, vol. 2012, 2012.

[39] A. Granas and J. Dugundji, Fixed Point Theory and Applications, Springer, New York, NY, USA, 2003. 